\title{
Pyrroline-5-carboxylate synthase senses cellular stress and modulates metabolism by regulating mitochondrial respiration
}

\author{
Zhaoying Yang ${ }^{1} \cdot$ Xiaocui Zhao ${ }^{1} \cdot$ Weina Shang ${ }^{1} \cdot$ Yang Liu $^{2} \cdot$ Jun-Feng $\mathrm{Ji}^{2} \cdot$ Jun-Ping $\mathrm{Liu}^{3} \cdot$ Chao Tong ${ }^{1,3,4}$
}

Received: 24 March 2020 / Revised: 10 July 2020 / Accepted: 23 July 2020 / Published online: 7 August 2020

(c) The Author(s), under exclusive licence to ADMC Associazione Differenziamento e Morte Cellulare 2020

\begin{abstract}
Pyrroline-5-carboxylate synthase (P5CS) catalyzes the synthesis of pyrroline-5-carboxylate (P5C), a key precursor for the synthesis of proline and ornithine. P5CS malfunction leads to multiple human diseases; however, the molecular mechanism underlying these diseases is unknown. We found that P5CS localizes in mitochondria in rod- and ring-like patterns but diffuses inside the mitochondria upon cellular starvation or exposure to oxidizing agents. Some of the human disease-related mutant forms of P5CS also exhibit diffused distribution. Multimerization (but not the catalytic activity) of P5CS regulates its localization. P5CS mutant cells have a reduced proliferation rate and are sensitive to cellular stresses. Flies lacking P5CS have reduced eclosion rates. Lipid droplets accumulate in the eyes of the newly eclosed P5CS mutant flies, which degenerate with aging. The loss of P5CS in cells leads to abnormal purine metabolism and lipid-droplet accumulation. The reduced lipid-droplet consumption is likely due to decreased expression of the fatty acid transporter, CPT1, and few $\beta$-oxidationrelated genes following P5CS knockdown. Surprisingly, we found that P5CS is required for mitochondrial respiratory complex organization and that the respiration defects in P5CS knockout cells likely contribute to the metabolic defects in purine synthesis and lipid consumption. This study links amino acid synthesis with mitochondrial respiration and other key metabolic processes, whose imbalance might contribute to P5CS-related disease conditions.
\end{abstract}

These authors contributed equally: Zhaoying Yang, Xiaocui Zhao

Edited by L. Scorrano

Supplementary information The online version of this article (https:// doi.org/10.1038/s41418-020-0601-5) contains supplementary material, which is available to authorized users.

Chao Tong

ctong@zju.edu.cn

1 MOE Key Laboratory for Biosystems Homeostasis and Protection and Innovation Center for Cell Signaling Network, Life Sciences Institute, Zhejiang University, Hangzhou, 310058 Zhejiang, China

2 Centre of Stem Cell and Regenerative Medicine, School of Medicine, Zhejiang University, Hangzhou, 310058 Zhejiang, China

3 Institute of Ageing Research, Hangzhou Normal University College of Medicine, Hangzhou, 311121, China

4 The Second Affiliated Hospital, School of Medicine, Zhejiang University, Hangzhou, 310009 Zhejiang, China

\section{Introduction}

Mitochondria is not only a power house to produce ATP but also a metabolic center involved in amino acid, fatty acid, and nucleotide metabolism in cells [1,2]. Malfunctions or declined quality and activity of mitochondria have been linked to many human diseases and aging [3, 4]. The crosstalk and coordination of multiple metabolic pathways in mitochondria, however, is less understood.

Pyrroline-5-carboxylate synthase (P5CS) is a mitochondrial-localized bifunctional enzyme that exhibits glutamate kinase (GK) and $\gamma$-glutamyl phosphate reductase (GPR) activities. P5CS catalyzes the synthesis of pyrroline5-carboxylate (P5C), a key precursor for the synthesis of proline and ornithine [5]. In humans, P5CS is encoded by the ALDH18A1 gene. Mutations of ALDH18A1 cause dominant (SPG9A) or recessive (SPG9B) spastic paraplegia, a degenerative neurological disorder that primarily affects upper motor neurons [6, 7]. In addition, ALDH18AI mutations have also been found in patients with autosomal dominant cutis laxa $(\mathrm{CL})$ with progeroid features ranging from joint laxity and skin hyper-elasticity to bilateral cataracts and progressive neurodegeneration [8-10]. 
Proline has been proposed to serve as an antioxidant to protect cells from oxidative stresses [11]. In addition, it is required for synthesis of brain polypeptides that might be required for neuroprotection [12]. Therefore, some of the symptoms associated with mutations of human ALDH18A1 can be explained by the lack of proline. However, the reduction of proline cannot account for all the disease symptoms. Furthermore, there is no obvious reduction of proline levels in some patients [10]. It remains unclear how the mutations in $A L D H 18 A 1$ lead to the disease conditions.

Here, we show that P5CS forms rod- and ring-like structures in mitochondria. It changes its distribution in response to the oxidative stress caused by starvation or other stimuli. The loss of P5CS caused defects in organization of mitochondrial respiratory complex and therefore led to abnormal lipid $\beta$-oxidation and purine metabolism.

\section{Results}

\section{P5CS forms rod- and ring-like structures in mitochondria}

In a study of mitochondrial proteins during aging, we came across an interesting observation that P5CS, a mitochondrial-localized enzyme, changed patterns in a drug-induced senescence cell model [13, 14]. In control IMR-90 cells, P5CS forms large bright puncta inside mitochondria. However, in cells induced to undergo senescence by treatment with doxorubicin (the characterization of cell senescence was shown in Fig. S1), the number of P5CS puncta increased and the size of the puncta decreased. Most strikingly, diffuse mitochondrial P5CS staining was commonly observed (Fig. 1a, a'). We wondered whether the level of P5CS changed in these senescent cells. However, western blot indicated there were no significant changes in P5CS levels (Fig. 1b, c), suggesting the altered P5CS pattern we observed was not due to changes in the level of protein expression.

We then decided to carefully examine the distribution of P5CS in multiple cell lines. In all the cell lines we tested (HeLa, 293T, and U2OS), P5CS showed punctate patterns, with the puncta distributed randomly in the mitochondria (Figs. 1d and S2). When examined with high-resolution microscopy, P5CS formed rod- and ring-like structures in mitochondria. The sizes of the rods or rings were variable (Fig. 1e). We then expressed a P5CS tagged with APEX at the C-terminus in HeLa cells and examined P5CS suborganelle localization by transmission electro-microscopy (TEM). As a control, the mitochondrial outer membrane TOMM20 was also tagged with APEX at the C-terminus and expressed in HeLa cells. TOMM20 ubiquitously marked the outer membrane of mitochondria (Fig. 1f), while
P5CS-APEX produced dark signals in the restricted areas inside mitochondria (Fig. 1g). Fractionation experiments indicated that P5CS was a mitochondrion-specific protein (Fig. 1h). In the Proteinase K (PK) treatment experiments, P5CS was degraded much more slowly than TOMM20, but showed a very similar degradation pattern as that of the matrix protein HSP60 (Fig. 1i). This suggests that P5CS is a matrix-localized protein. We then examined whether P5CS could integrate into the mitochondrial membrane using alkaline treatments. The majority of P5CS was released into the supernatant upon high-pH buffer treatment (Fig. 1j), indicating that it is not a membrane-integrated protein.

\section{The dimerization domains are required for P5CS puncta patterns}

Point mutations in P5CS are linked to multiple diseases including CL, a disease with multiple premature ageing features. One of the disease-related mutants of P5CS, Arg138Gln, was reported to have a diffused distribution in mitochondria [10]. We then examined whether other disease-related P5CS point mutations also led to altered P5CS distribution. We expressed V5-tagged wild-type or disease-related mutant forms of P5CS in HeLa cells and examined their distribution. Similar to endogenous P5CS, the overexpressed wild-type P5CS also formed distinct puncta inside mitochondria. Most disease-related P5CS mutants such as $\mathrm{P}^{2} \mathrm{CS}^{\mathrm{S} 742 \mathrm{I}}, \mathrm{P} \mathrm{CS}^{\mathrm{R} 765 \mathrm{Q}}, \mathrm{P}^{2} \mathrm{CS}^{\mathrm{H} 784 \mathrm{Y}}$, $\mathrm{P} \mathrm{CS}^{\mathrm{R} 425 \mathrm{C}}$, and $\mathrm{P} 5 \mathrm{CS} \mathrm{S}^{\mathrm{R} 749 \mathrm{Q}}$ also have similar distributions as that of the wild-type (Fig. 2a-f). However, three diseaserelated point mutations $\left(\mathrm{P}^{2} \mathrm{CS}^{\mathrm{R} 84 \mathrm{Q}}, \mathrm{P} \mathrm{CS}^{\mathrm{G} 93 \mathrm{R}}\right.$ and P5CS ${ }^{\mathrm{R} 138 \mathrm{Q}}$ ) exhibited a diffuse staining pattern inside mitochondria in a proportion of cells following overexpression (Fig. 2g-i'). Among them, P5CS ${ }^{\mathrm{R} 138 \mathrm{Q}}$ was diffused in $\sim 91.9 \%$ of cells following overexpression. It has been reported that P5CS can form homodimers/multimers. We therefore wondered whether $\mathrm{P} 5 \mathrm{CS}^{\mathrm{R} 138 \mathrm{Q}}$ could form dimers with wild-type P5CS (P5CS ${ }^{\mathrm{WT}}$ ) and affect its distribution. Indeed, $\mathrm{P} 5 \mathrm{CS}^{\mathrm{R} 138 \mathrm{Q}}$ could bind to $\mathrm{P} 5 \mathrm{CS} \mathrm{ST}^{\mathrm{WT}}$ (Fig. 2k). When they were co-expressed, P5CS ${ }^{\mathrm{R} 138 \mathrm{Q}}$ and $\mathrm{P} \mathrm{CS}^{\mathrm{WT}}$ were co-localized and showed three different patterns in cells: diffused in mitochondria (similar to $\mathrm{P} \mathrm{CS}^{\mathrm{R} 138 \mathrm{Q}}$ expression alone, $38 \%$ ); puncta in mitochondria (similar to $\mathrm{P} 5 \mathrm{CS}^{\mathrm{WT}}$ expression alone, 11\%), and a diffused pattern with some puncta $(51 \%)$ (Fig. $\left.2 \mathrm{j}-\mathrm{j}^{\prime \prime}\right)$. These data indicate that $\mathrm{P} 5 \mathrm{CS}^{\mathrm{R} 138 \mathrm{Q}}$ can dominantly affect the distribution of $\mathrm{P} 5 \mathrm{CS}{ }^{\mathrm{WT}}$.

Mammalian P5CS is a bifunctional enzyme that exhibits GK and GPR activities. We then wondered whether the enzyme activities were required for its proper distribution. To test this, we mutated the potential enzyme activity centers individually or together and examined their distribution. We mutated three main residues (Lys76, Asp247, 

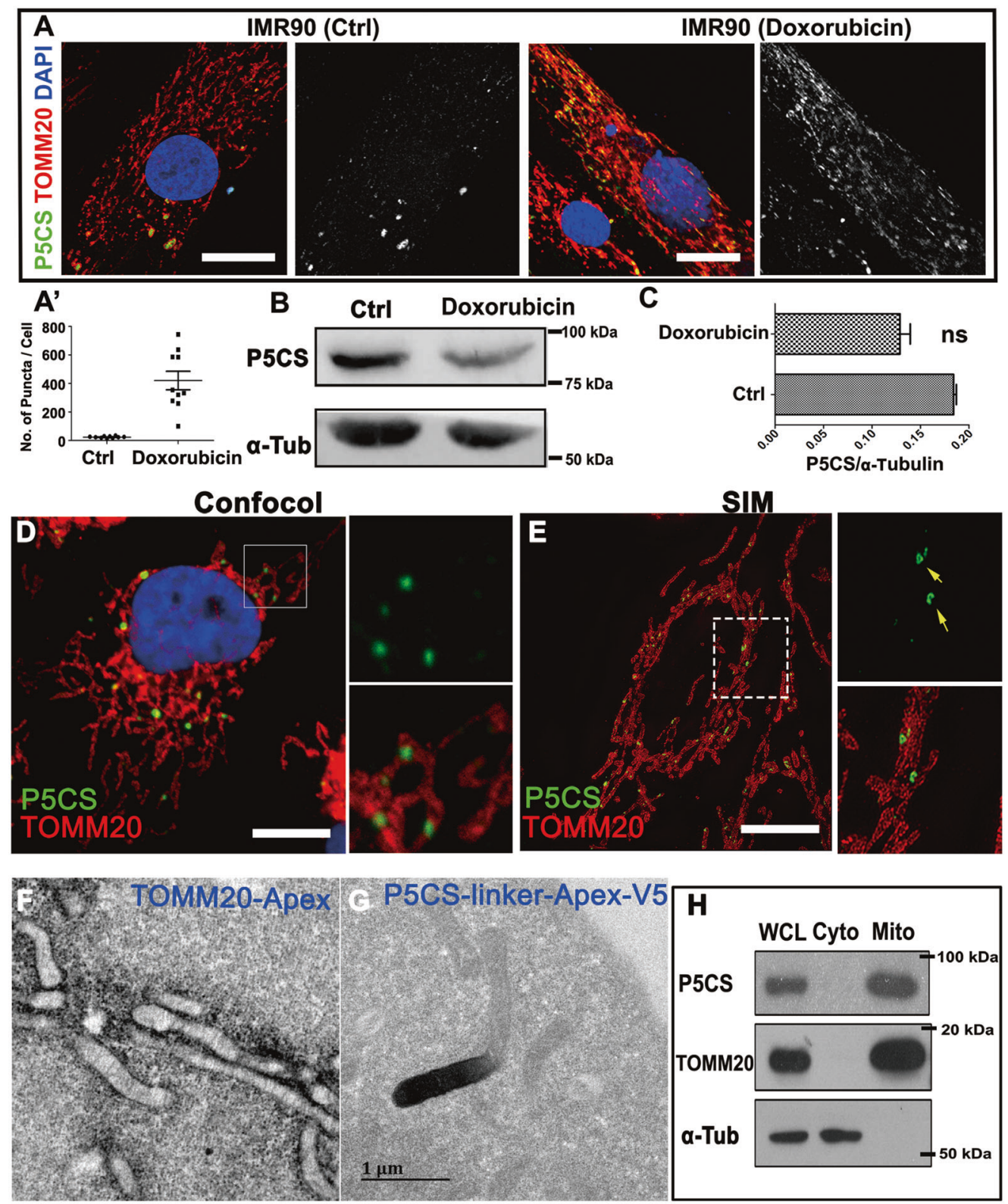

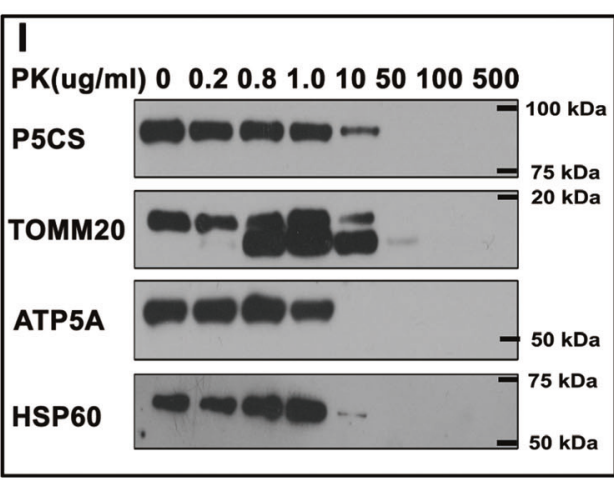

Lys311) of the activity center of the GK moiety $[15,16]$, made a V5-tagged mutated form of P5CS: P5CS ${ }^{\text {K76A-D247A- }}$ $\mathrm{K} 311 \mathrm{~A}$ and expressed it in cells. The catalytic cysteine

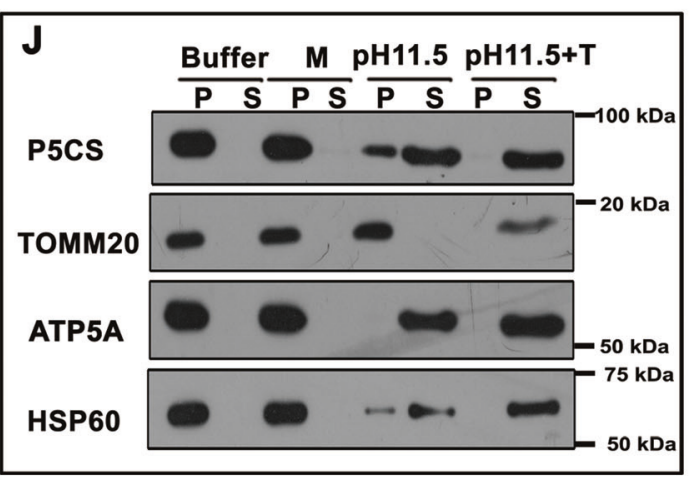

(Cys612) of the GPR moiety [17, 18] was also mutated to alanine. None of these mutations changed the P5CS localization pattern (Fig. 2q-s). These data indicate that the 
Fig. 1 P5CS forms rod- and ring-like structures in mitochondria. a Control (Ctrl) and Doxorubicin-induced senescent IMR-90 cells were fixed and stained with anti-P5CS (green) and anti-TOMM20 (red) antibodies. In control IMR-90 cells, P5CS forms large bright puncta inside mitochondria. In cells induced to undergo senescence by treatment with doxorubicin, P5CS became diffused. $\mathbf{a}^{\prime}$ is a quantification of the P5CS puncta numbers per cell in the cells with indicated treatments. b, c The expression levels of P5CS in Ctrl and senescent IMR-90 cells were comparable. $\alpha$-tubulin served as the loading control. c Statistical analysis of $\mathbf{b}$. Data were presented as mean + SEM, $n=3$. d Confocal image of HeLa cells stained with anti-P5CS (green) and anti-TOMM20 (red) antibodies indicates that P5CS forms puncta inside the mitochondria. e SIM image of HeLa cells stained with antiP5CS (green) and anti-TOMM20 (red) antibodies indicates that P5CS localizes in mitochondria with a rod and ring-like pattern. $f$ TEM image of HeLa cells expressing mitochondrial outer membrane protein TOMM20 tagged with Apex at the C-terminus. DAB reaction product was restricted to the surface of mitochondria. g TEM image of HeLa cells expressing P5CS tagged with Apex at the C-terminus. DAB reaction product was restricted to the matrix of mitochondria. $\mathbf{h}$ Cell fractionation assay of HeLa cells indicates that endogenous P5CS was enriched in a mitochondria fraction positive for TOMM20. WCL whole cell lysate, Cyto cytoplasm, Mito mitochondrion. i PK digestion experiments show that endogenous P5CS has similar digestion patterns to those of the mitochondrial matrix protein HSP60. $\mathbf{j}$ Alkaline carbonate extraction assay shows that majority of P5CS, ATP5A, and HSP60 could be released to the supernatant with $\mathrm{pH} 11.5$ sodium carbonate buffer treatment, while membrane-integrated protein TOMM20 cannot be released. M: purified mitochondria suspended in isotonic buffer; buffer: mock-treated buffer; $\mathrm{pH}$ 11.5: $\mathrm{pH} 11.5$ sodium carbonate buffer; $\mathrm{pH} 11.5+\mathrm{T}$ : $\mathrm{pH} 11.5$ sodium carbonate buffer with Triton X-100; P: pellet; S: supernatant. Scale bar: $10 \mu \mathrm{m}$.

enzymatic activities of P5CS are not required for its specific mitochondrial localization patterns.

P5CS protein includes a mitochondrial-targeting domain, a GK moiety, and a GPR moiety. The GPR moiety includes a cofactor binding domain, a catalytic domain, and two regions required for oligomerization (Fig. 21) [5]. To analyze which domain is required for the specific distribution of P5CS, we made several truncation forms of P5CS and expressed them in HeLa cells. The loss of a potential mitochondrial-targeting domain together with the GK moiety resulted in a ubiquitous cytoplasmic distribution of P5CS (Fig. 2p). The lack of oligomerization regions leads to a diffuse mitochondrial distribution of P5CS (Fig. 2m-o), indicating that the oligomerization domains are required for the rod- and ring-like distribution of P5CS.

\section{P5CS diffuses throughout the mitochondria upon oxidative stress}

We then wanted to examine whether P5CS localization was affected by exogenous stimulation. Interestingly, we found that P5CS became diffuse when the cells were treated with HBSS (Figs. 3a-d and S3A). There was no significant change in P5CS mRNA and protein levels in this condition
(Fig. S3B-D). We next attempted to identify which nutrient was the critical determinant of the normal P5CS distribution. Serum starvation (Fig. 3a-d) or low glucose (Fig. 3a-d) did not change the pattern of P5CS. However, P5CS diffused throughout the mitochondria upon amino acid starvation (Fig. 3a-d) or following culture in medium without glutamine (Fig. 3a-d). To test whether the change of distribution is due to the induction of autophagy, we incubated the cells with HBSS and the autophagy inhibitor bafilomycin A. The block of autophagy did not inhibit HBSS-induced P5CS relocalization (Fig. 3a-d). Surprisingly, adding the ROS scavenger NAC to HBSS suppressed HBSS-induced P5CS relocalization (Fig. 3a-d). As reported previously, ROS levels increased in cells cultured in HBSS [19]. MitoSOX staining indicated that the levels of ROS in mitochondria were indeed increased with the treatment of HBSS, while adding NAC partially suppressed it (Fig. S3E, F). However, reducing mitochondrial ROS with MitoTEMPO did not significantly inhibit HBSS-induced P5CS relocalization (Figs. 3a-d and S3E). Mito-TEMPO is a mitochondrial-enriched SOD mimetic that dismutases superoxide to form $\mathrm{H}_{2} \mathrm{O}_{2}$, while NAC scavenges peroxynitrite and $\mathrm{H}_{2} \mathrm{O}_{2}$ by promoting Glutathione (GSH) production. The different ROS elimination mechanisms used by Mito-TEMPO and NAC might contribute to the different effects of these two antioxidants to HBSS-induced P5CS relocalization.

To test whether the increase of $\mathrm{H}_{2} \mathrm{O}_{2}$ was required for altered P5CS localization, we treated the cells with $\mathrm{H}_{2} \mathrm{O}_{2}$. Indeed, P5CS became diffused throughout mitochondria in these cells (Fig. 3a-d). GSH is the key ROS scavenger in cells [20]. We wondered whether reducing the synthesis of GSH would also alter P5CS patterns. We knocked down the catalytic subunit of glutamate cysteine ligase (GCLC), the rate-limiting enzyme, or GSH synthetase (GSS), the second enzyme in the GSH biosynthesis pathway in HeLa cells and examined P5CS localization. Indeed, P5CS became diffused in mitochondria when GCLC or GSS was reduced (Fig. 3a-d). The P5CS distribution also became diffuse when we decreased levels of the reduced form of GSH by knocking down GSH reductase (GSR) with siRNA (Fig. 3a-d). All these data suggest that oxidative stimuli engender changes in P5CS staining patterns.

HBSS treatment increased fatty acid $\beta$-oxidation in mitochondria [21], which in turn increased mitochondrial ROS production. Treatment of cells with HBSS together with Etomoxir (a carnitine palmitoyl transferase 1 (CPT1) inhibitor that inhibits fatty acid mitochondrial import), HBSS-induced increasing of ROS was suppressed and P5CS relocalization was inhibited (Figs. 3a-d and S3E, F). Similar to the drug treatment, when we knocked down CPT1A with siRNA (Fig. 3a-d), CPT2 (Fig. 3a-d), or 

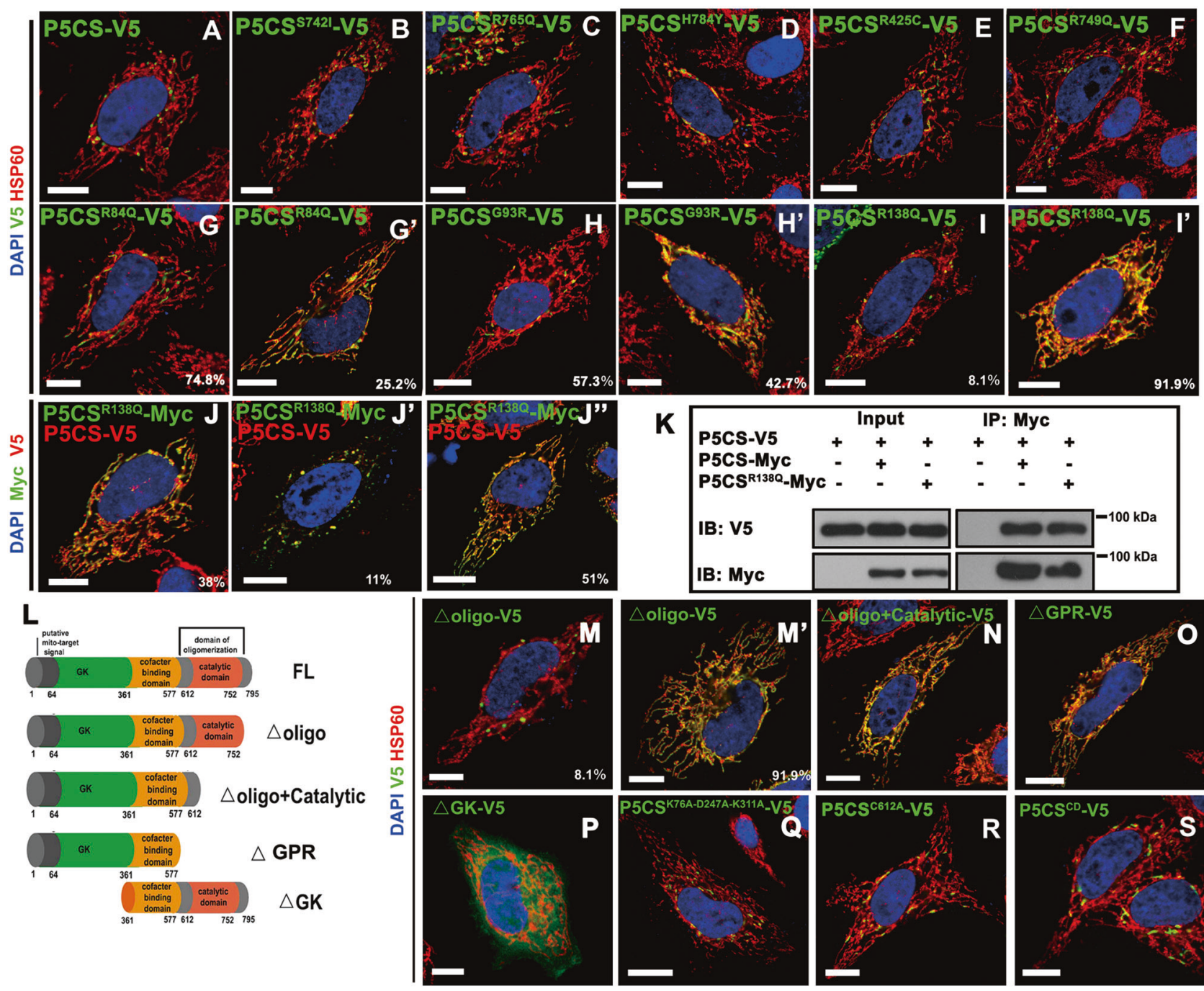

Fig. 2 The dimerization domains are required for P5CS puncta patterns. a-i' $\mathbf{i}^{\prime}$ HeLa cells expressing V5-tagged wild-type or diseasesrelated mutant forms of P5CS were fixed and stained with anti-V5 and anti-HSP60 antibody. For some mutant forms of P5CS, two different distribution patterns (puncta or diffused) were observed. The ratios of cells with typical P5CS distribution patterns were labeled at the bottom of images. $\mathbf{j}-\mathbf{j}^{\prime \prime}$ HeLa cells expressing Myc-tagged P5CS ${ }^{\mathrm{R} 138 \mathrm{Q}}$ and V5 tagged wildtype P5CS together were fixed and stained with anti-Myc (green) and anti-V5 (red) antibody. Three different patterns were observed: diffused in mitochondria (similar to $\mathrm{P} 5 \mathrm{CS}^{\mathrm{R} 138 \mathrm{Q}}$ expression alone, $38 \%$ ); puncta in mitochondria (similar to $\mathrm{P} 5 \mathrm{CS}^{\mathrm{WT}}$ expression alone, $11 \%$ ), and a diffused pattern with some puncta (51\%). k Co-IP experiments showed that both P5CS-Myc and P5CS ${ }^{\mathrm{R} 138 \mathrm{Q}}-\mathrm{Myc}$ could

SLC25A20 (the carnitine-acylcarnitine translocase; Fig. 3a-d), HBSS-induced P5CS relocalization was also blocked. The ACAD9 enzyme catalyzes a crucial step in fatty acid $\beta$-oxidation by forming a $\mathrm{C} 2-\mathrm{C} 3$ trans-double bond in the fatty acid. ACADL is one of the four enzymes that catalyze the initial step of mitochondrial $\beta$-oxidation of straight-chain fatty acids. HBSS-induced P5CS relocalization was also partially blocked when ACAD9 or ACADL was knocked down (Fig. 3a-d). pull down P5CS-V5 when they were co-expressed in Hela cells. I A schematic diagram indicated the domain organization of human P5CS and the truncation forms that were made in this study. $\mathbf{m}-\mathbf{p}$ HeLa cells expressing the indicated V5-tagged truncation forms of P5CS were fixed and stained with anti-V5 and anti-HSP60 antibody. For the truncation forms of P5CS, which have two different distributions, the ratio of cells with typical P5CS distribution patterns was labeled at the bottom of images. q-s HeLa cells expressing the enzyme activity dead forms of P5CS (P5CS ${ }^{\mathrm{CD}}$ with K76, D247, K311, and C612 mutated to A) were fixed and stained with anti-V5 and anti-HSP60 antibody. Loss of catalytic activity did not affect P5CS distribution patterns. Scale bar: $10 \mu \mathrm{m}$.

\section{Loss of P5CS leads to increased sensitivity to stress and neurodegeneration}

Since P5CS redistributes upon stimulation, we hypothesized that it might sense stress and modulate cellular activities. To test that, we made a P5CS knockout (KO) cell line using CRISPR/Cas9 and examined the function of P5CS in cells. A single nucleotide deletion led to a premature stop codon and the loss of P5CS protein (Fig. 4a, b). The proliferation 


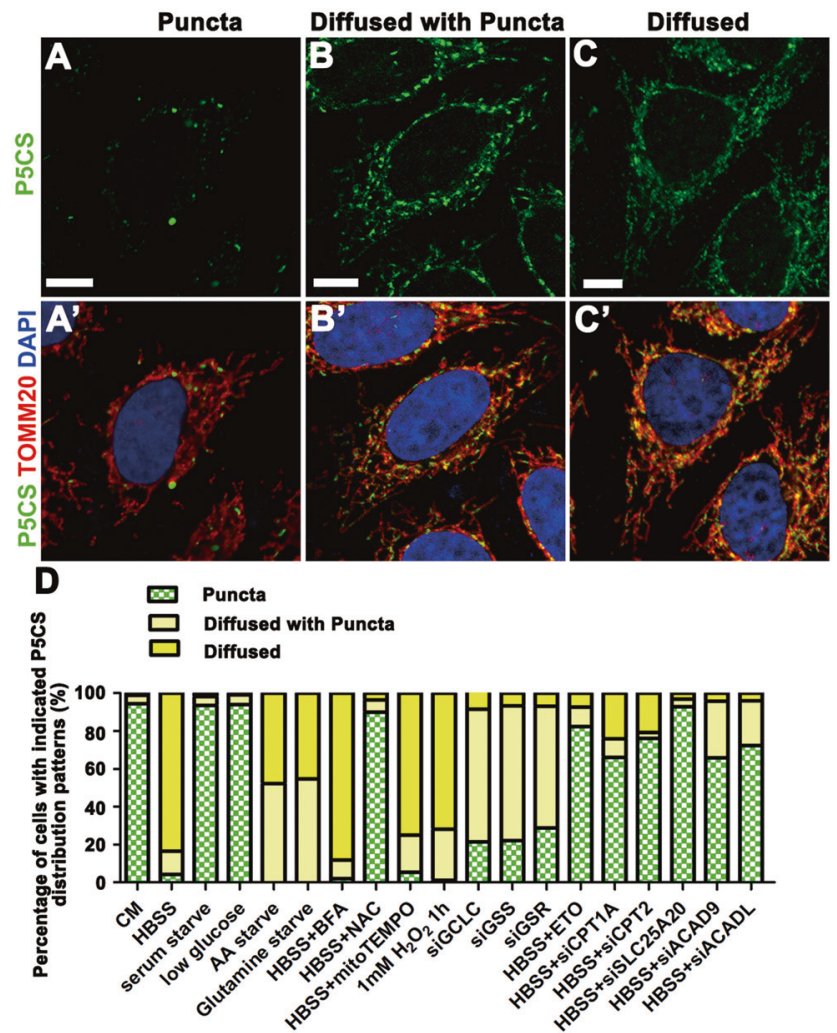

Fig. 3 P5CS diffuses throughout the mitochondria upon oxidative stress. Hela cells with indicated treatments $(\mathbf{d})$ were fixed and stained with anti-P5CS (green) and anti-TOMM20 (red) antibodies. DAPI (blue) staining indicates the nuclei. a-c $\mathbf{c}^{\prime}$ The typical distributions of P5CS were shown. a, $\mathbf{a}^{\prime}$ Puncta. $\mathbf{b}, \mathbf{b}^{\prime}$ Diffused with puncta. $\mathbf{c}, \mathbf{c}^{\prime}$ Diffused. a-c showed the green channels (P5CS). $\mathbf{a}^{\prime}-\mathbf{c}^{\prime}$ showed the merged channels. $\mathbf{d}$ is the quantification of the proportions of cells with different P5CS patterns when the cells were treated with indicated treatments. CM: complete medium; AA: amino acid; si: RNAi knockdown. Scale bar: $10 \mu \mathrm{m}$.

rate of P5CS KO cells was lower than that in the control cells (Fig. 4c). Under HBSS starvation conditions, P5CS $\mathrm{KO}$ cells showed an even more dramatic reduction of proliferation rate than that of control cells (Fig. 4d), suggesting that loss of P5CS increases cell sensitivity to stress.

To test the in vivo activity of P5CS, we made $C G 7470$ $(d P 5 C S)$ KO flies using CRISPR/Cas9. Three mutants, $d P 5 C S^{67}, d P 5 C S^{73}$, and $d P 5 C S^{74}$, were recovered. All these mutants were predicted to encode proteins with premature stop codons (Fig. 4e). Since all three mutant lines have similar phenotypes, we studied $d P 5 C S^{67}$ in detail. $d P 5 C S^{67}$ flies were semi-lethal with an eclosion rate of $\sim 50 \%$ (Fig. 4f). The eyes of surviving flies were examined at day 1 and day 30 by TEM (Fig. $4 \mathrm{~g}-\mathrm{h}$ ). At day 1, the retina of $d P 5 C S^{67}$ flies had grossly normal morphology. One striking feature was that the glia cells were swollen and accumulated many lipid droplets (LDs) (Fig. 4g, i), a defect often reported in flies with defects in lipid metabolism [22]. At day 30 , there was a significant reduction in the number of photoreceptor cells in $d P 5 C S^{67}$ flies when compared with wild-type controls, suggesting P5CS is required for neuronal homeostasis.

\section{Loss of P5CS leads to multiple metabolic defects}

To understand changes in metabolic networks upon the loss of P5CS, we performed a quasi-target metabolomics analysis of controls and P5CS KO cells. Among 420 metabolites identified in the analysis, the levels of 79 were significantly different between control and P5CS KO cells (Figs. S4, S5 and Tables S1, S2). Not surprising, six metabolites were related to proline synthesis and the urea cycle was upregulated in the P5CS KO cells (Fig. 5a, d), probably due to the direct effects of the lack of P5CS enzyme activity. Unexpectedly, but consistent with the accumulation of LDs in fly eyes, we found that several intermediate metabolites of $\beta$-oxidation were increased in P5CS KO cells (Fig. 5b). In addition, the levels of many nucleotides and their derivatives were changed (Fig. 5c), indicating that purine metabolism was affected in P5CS KO cells. Indeed, when we expressed phosphoribosyl formylglycinamidine synthase (FGAMS)-mEos to label purinosomes, we found that the loss of P5CS increased the number of purinosome-positive cells under normal medium culture conditions (Fig. 5e, f).

LD accumulation was observed in CL patient fibroblasts. The accumulation of LDs in fly eyes and the accumulation of intermediate metabolites of fatty acid $\beta$-oxidation in P5CS KO cells indicated that the loss of P5CS might affect lipid metabolism. We therefore knocked down P5CS in multiple cell lines and examined LDs by BODIPY staining. The reduction of P5CS indeed increased LDs in the cells (Fig. $5 \mathrm{~g}-\mathrm{k}$ ). Although P5CS is a mitochondrial-localized enzyme, TEM analysis indicated that mitochondrial morphology did not change significantly upon the reduction of P5CS (Fig. 5j"). P5CS KO cells also accumulated large amount of LDs (Fig. 5m, o), which could be rescued by introduction of wild-type P5CS into the KO cells (Fig. 5n, o). Interestingly, overexpression of $\mathrm{P}^{2} \mathrm{CS}^{\mathrm{R} 138 \mathrm{Q}}$, but not wild-type P5CS, led to accumulation of LDs (Fig. 5p-s), suggesting that $\mathrm{P} 5 \mathrm{CS}^{\mathrm{R} 138 \mathrm{Q}}$ functions in a dominant negative manner to regulate lipid metabolism. These data suggest that P5CS KO not only affects proline synthesis and the urea cycle, but also leads to abnormal fatty acid $\beta$-oxidation and purine metabolism.

\section{P5CS loss inhibits fatty acid $\beta$-oxidation}

P5CS-induced LD accumulation could be due to increased lipid synthesis or reduced lipolysis and fatty acid $\beta$ oxidation. To distinguish between these possibilities, we performed experiments to measure lipid flux. DGAT1/2 are 
Fig. 4 Loss of P5CS leads to increased sensitivity to stress and neurodegeneration. a A schematic diagram indicated the CRISPR/Cas9 knockout strategy in cultured HeLa cells. The gRNA targeting human ALDH18A1 was underlined. The indel of the isolated knockout (KO) cell line was indicated. Position of the premature stop codon resulted from the indel was indicated in the diagram. b Western blot confirmed the loss of P5CS protein from the KO cells. Ctrl: control. c The proliferation rates of the control cells (Ctrl) and P5CS KO (KO) cells in complete medium (CM) were measured and normalized by dividing the cell numbers at indicated time points to the cell numbers at $0 \mathrm{~h}$. d The proliferation rates of Ctrl and P5CS KO cells in HBSS were measured and normalized. Data were presented as mean $+\mathrm{SEM}$, $n=3$. e A schematic diagram indicated the CRISPR/Cas9 knockout strategy in flies. The gRNA targeting $C G 7470$ $(d P 5 C S)$ was underlined. The indels of the isolated strains was indicated. Positions of the premature stop codon resulted from the indels were indicated in the diagram. $\mathbf{f}$ The eclosion rates of the heterozygous and homozygous $d P 5 C S^{67}$ were measured. The data was presented as mean \pm SEM, $n=$ 10 groups, each group contains at least 30 animals. g The retina of the wild-type (WT) and homozygous $d P 5 C S^{67}$ mutant flies were analyzed by TEM at day 1 and day 30 . $\mathbf{h}$ The numbers of the rhabdomeres per ommatidia were counted and plotted. The 30 days old $d P 5 C S^{67}$ mutant flies have reduced rhabdomeres than the age-matched controls. i The numbers of lipid droplets per ommatidia in 1-day-old WT and homozygous $d P 5 C S^{67}$ mutant flies were quantified. The data were presented as mean $+\mathrm{SEM}$, $n=30$.
A

ALDH18A1 gRNA-indels

CCAAGAGAATCGTGGTGAAGCTCGGCAGTGCCGTGGTGACOCGAGG Ctrl CCAAGAGAATCG GGTGAAGCTCGGCAGT - CCGTGGTGACCCGAGG[-1] P5CS KO
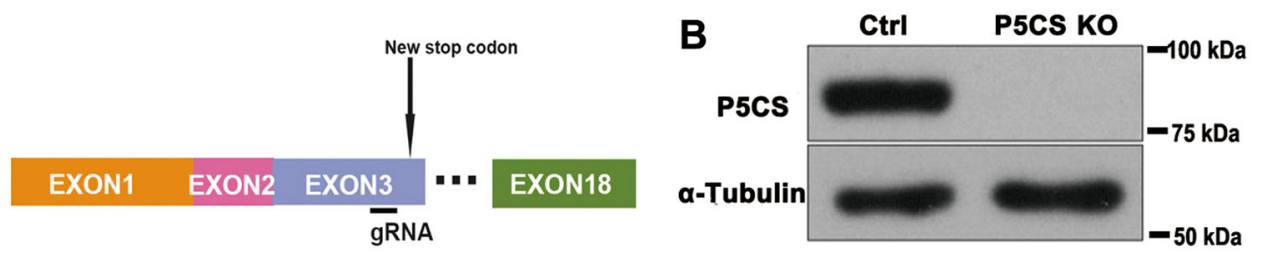

C
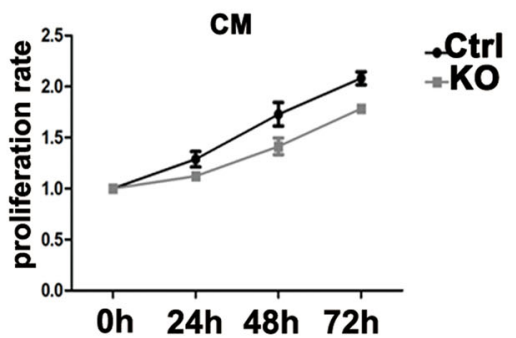

E
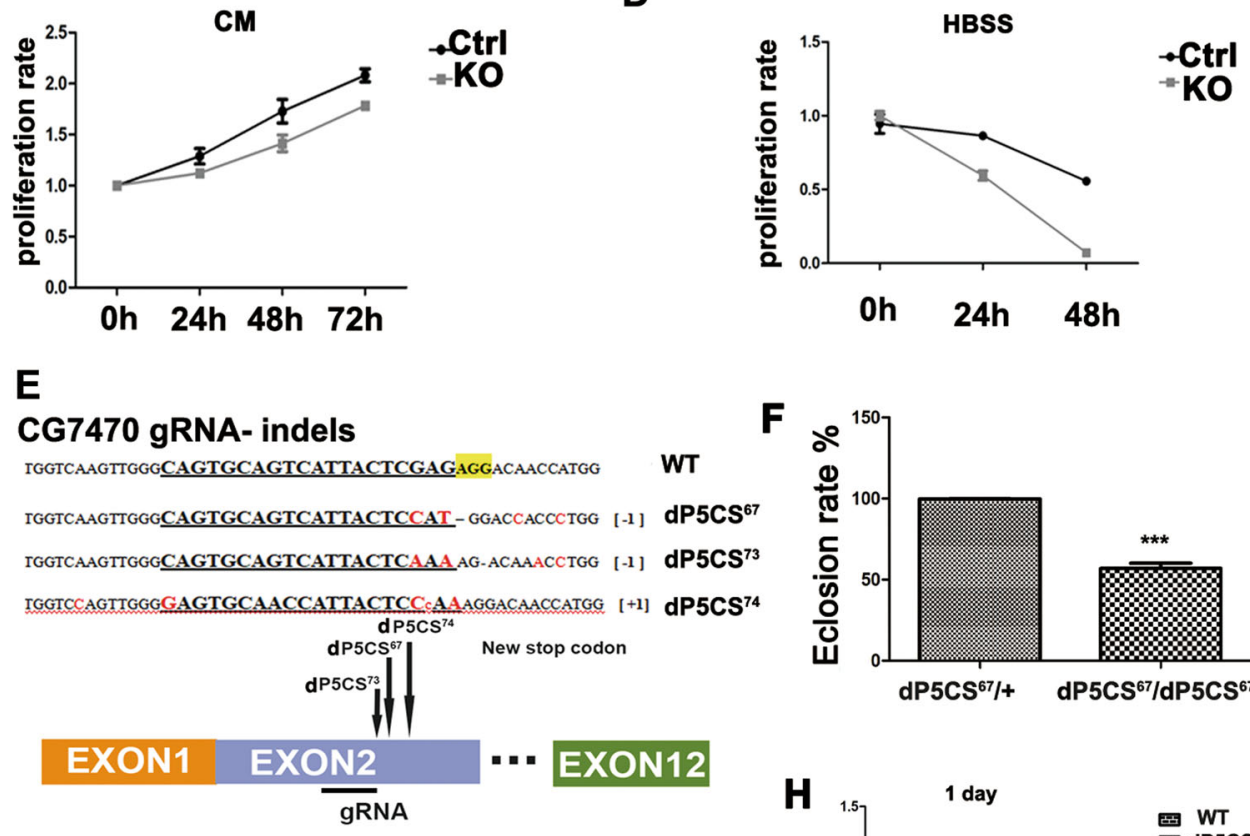

G

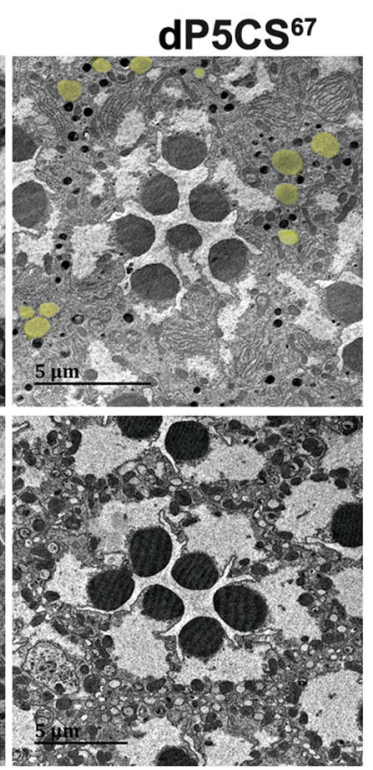

H

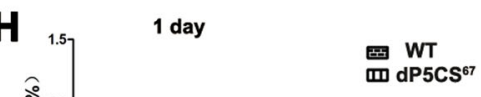

enzymes required for TAG synthesis from DAG [23, 24] (Fig. 6a). We knocked down P5CS expression and added DGAT1 and DGAT2 inhibitors after $24 \mathrm{~h}$ and then monitored LDs by BODIPY staining at different time points after the drug treatment (Fig. 6b). The addition of DGAT1/2 inhibitors blocked TAG synthesis and new LD formation, 

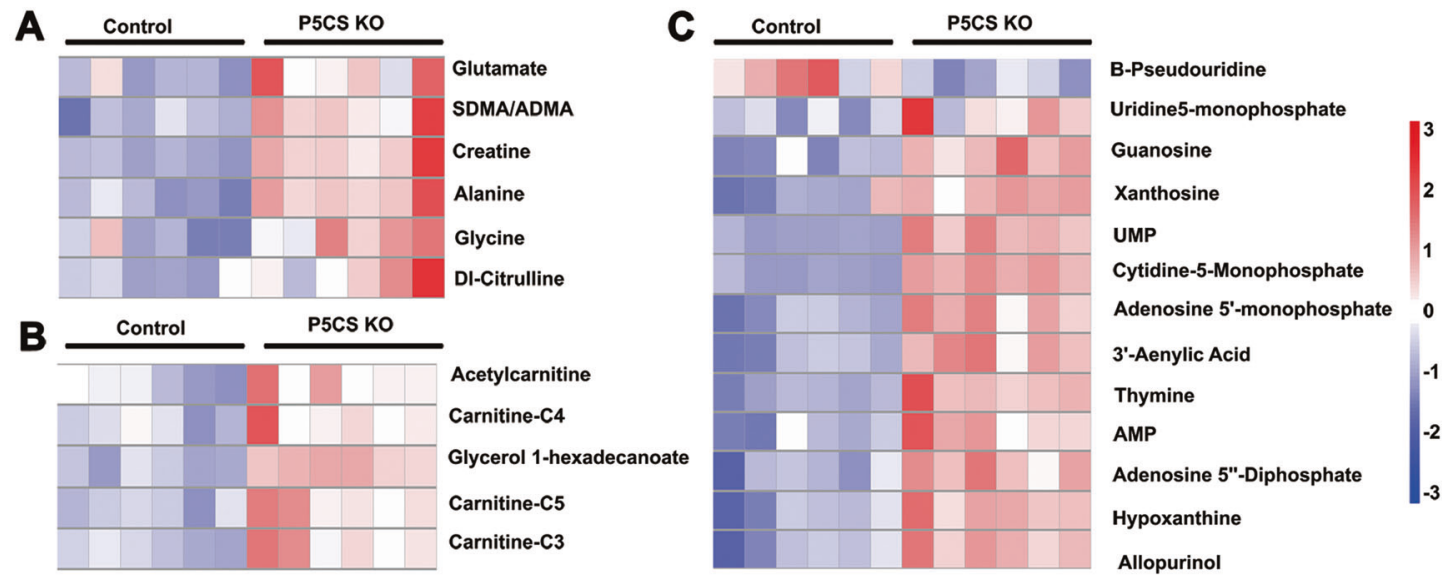

D
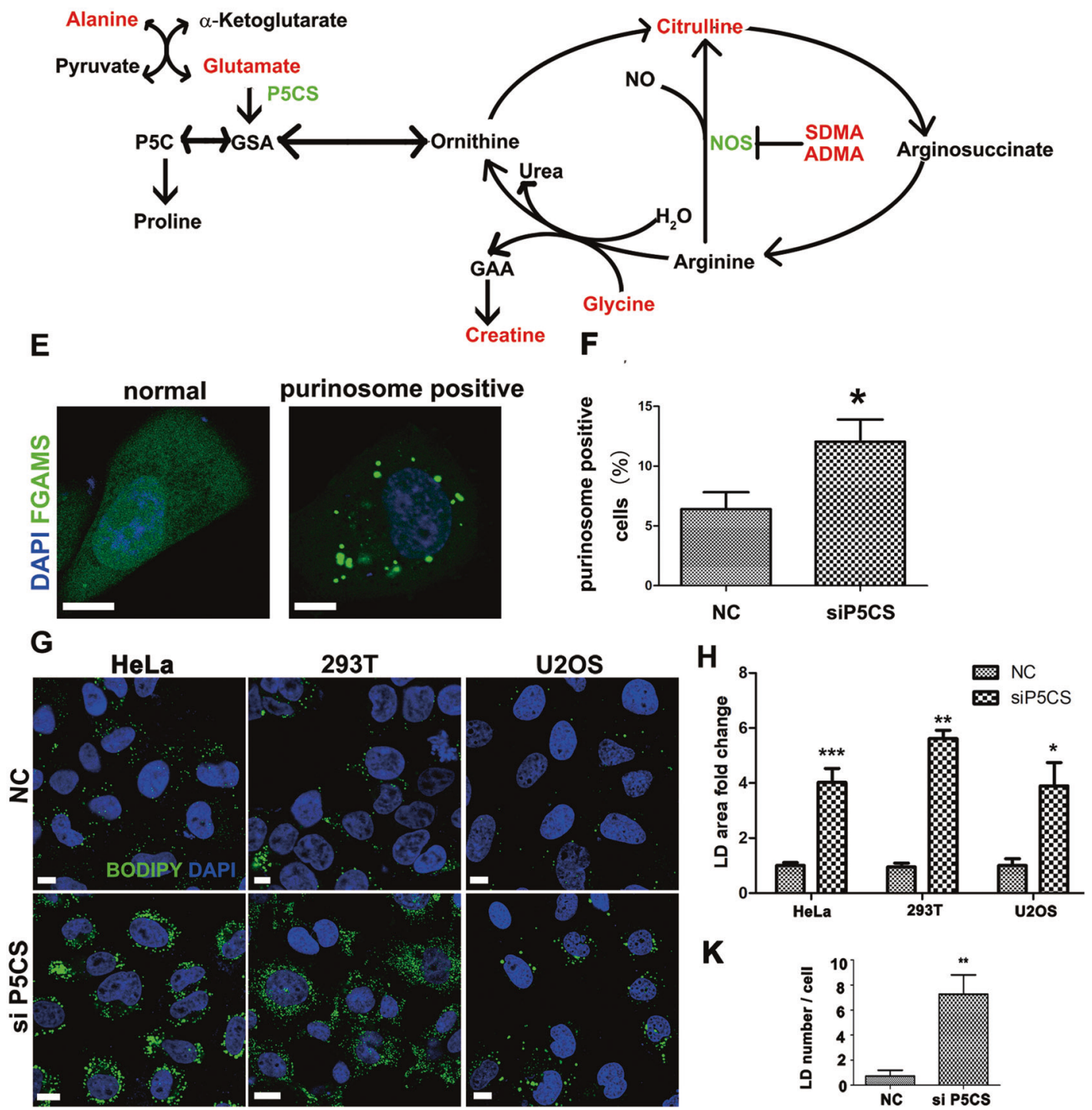

$\mathbf{K}$

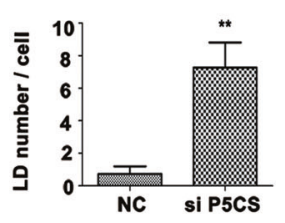

Fig. 5 (continued)

demonstrating that we could measure the consumption of LDs. With DGAT1/2 inhibitors treatment, the amount of LDs was greatly reduced in the control cells in $10 \mathrm{~h}$, while the reduction of LDs in the P5CS RNAi cells was not obvious (Figs. 6c and S6A, B). Similar results were observed for the P5CS KO cells (Fig. S6C, D). These data 

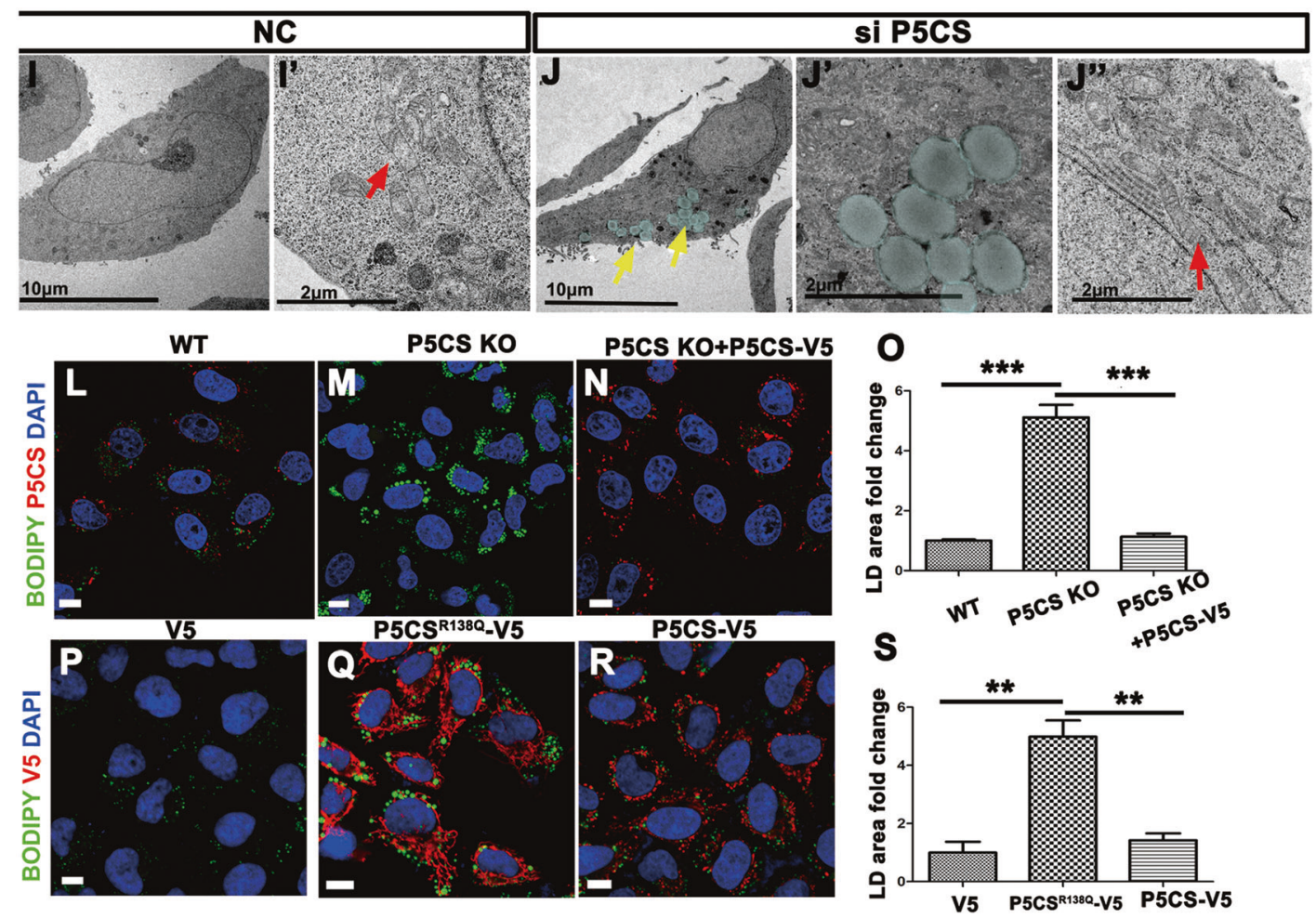

Fig. 5 Loss of P5CS leads to multiple metabolic defects. A quasitarget metabolomics analysis of controls and P5CS KO cells was performed. Six independent samples for each genotype were analyzed and the data were presented as heat map. Upregulated and downregulated metabolites were indicated by red and blue hues, respectively. Color intensity indicates the expression levels of metabolites as displayed. a Six metabolites were related to proline synthesis and the urea cycle was upregulated in the P5CS KO cells. b Five intermediate metabolites of $\beta$-oxidation were increased in P5CS KO cells. c The levels of 13 nucleotides and their derivatives were changed in P5CS KO cells. d A diagram showed the metabolic pathways of proline synthesis and urea cycle. e, f (FGAMS)-mEos was expressed in Hela cells subjected to control and P5CS RNAi treatments. The loss of P5CS increased the number of purinosome-positive cells under normal medium culture conditions. More than 300 cells from three independent samples were quantified. g P5CS was RNAi knockdown in HeLa, 293T, and U2OS cells. Lipid droplets were stained by BODIPY. The reduction of P5CS increased lipid droplets in the cells. $\mathbf{h}$ The quantification of the fold changes of LD area upon P5CS RNAi in three different cell types. Data were presented as mean $+\mathrm{SEM}, n=5$

suggest that the consumption of LDs is decreased in P5CSdeficient cells. To test whether the production of LDs is increased or not, we performed a pulse-chase experiment. We treated the cells with DGAT1/2 inhibitors when RNAi knockdown was initiated, and $24 \mathrm{~h}$ later we washed off the inhibitors and added an inhibitor of ATGL (ATGListatin) [25], the key enzyme required for lipolysis (Fig. 6a). This treatment blocks the consumption of LDs and allowed us to measure the number of LDs at different time points. Addition of DGAT1/2 inhibitors at the beginning blocked LD formation. Once P5CS levels were reduced, DGAT1/2 inhibitors were washed off and LDs started to form; images (more than 100 cells for each genotype were counted). $\mathbf{i}-\mathbf{j}^{\prime \prime}$ TEM analysis of the control HeLa cells and HeLa cells $\left(\mathbf{i}, \mathbf{i}^{\prime}\right)$ with P5CS RNAi knockdown $\left(\mathbf{j}, \mathbf{j}^{\prime \prime}\right)$. Mitochondrial morphology did not change significantly upon the reduction of P5CS $\left(\mathbf{i}^{\prime}, \mathbf{j}^{\prime \prime}\right)$. Red arrows indicated mitochondria. P5CS KO cells accumulated large amount of lipid droplets $\left(j, j^{\prime}\right)$. $\mathbf{k}$ The quantification of the numbers of LDs per cell in the TEM analysis. Data were presented as mean $+\mathrm{SEM}, n=$ 11 cells. I-n LDs were accumulated in P5CS KO cells, which could be rescued by introduction of wild-type P5CS into the KO cells. $\mathbf{o}$ is the quantification of the fold changes of LD area in the cells with indicated genotypes. Data were presented as mean $+\mathrm{SEM}, n=5$ groups (more than 100 cells for each genotype were counted). p-r HeLa cells transfected with V5 tag empty vector (V5), P5CS ${ }^{\mathrm{R} 138 \mathrm{Q}}$-V5, or P5CSV5 were stained with anti-V5 antibody and BODIPY. Overexpression of, P5CS ${ }^{\mathrm{R} 138 \mathrm{Q}_{-} \mathrm{V} 5}$ but not P5CS-V5 led to accumulation of lipid droplets. $\mathbf{S}$ is the quantification of the fold changes of LD area in the cells with indicated genotypes. Data were presented as mean + SEM, $n=5$ groups (more than 100 cells for each genotype were counted). Scale bar for the immunofluorescence images: $10 \mu \mathrm{m}$.

meanwhile the consumption of LDs was blocked (Fig. $6 b^{\prime}$ ). By measuring the amount of LDs at different time points, we could estimate the rate of LD formation. Although the cells with reduced P5CS have slightly more LDs than control cells, the rates of LD formation were largely the same in the control and P5CS knockdown cells (Figs. $6 \mathrm{c}^{\prime}$ and S6E, F). These data suggest that LD consumption (but not formation) was defective in P5CS knockdown cells.

We then performed RNA seq to examine gene expression in control and P5CS knockdown cells (Table S3). The expression of many genes related to fatty 
A

A

TAG synthesis

B +DGAT1i DGAT2i B

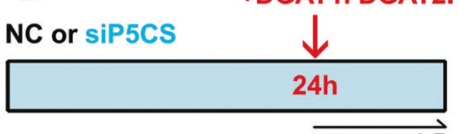

B' +DGAT1i DGAT2i wash off DGAT1i/2i
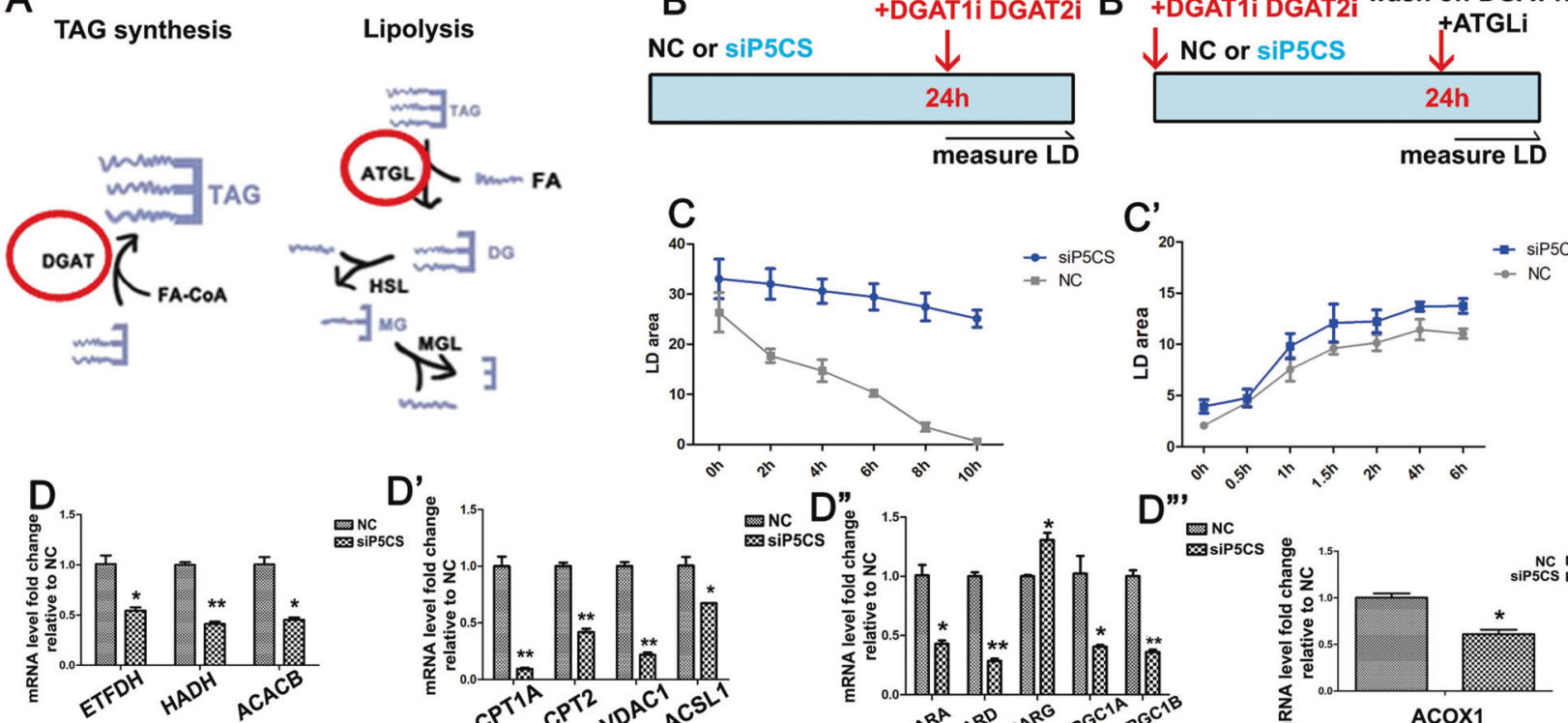

measure LD

measure LD
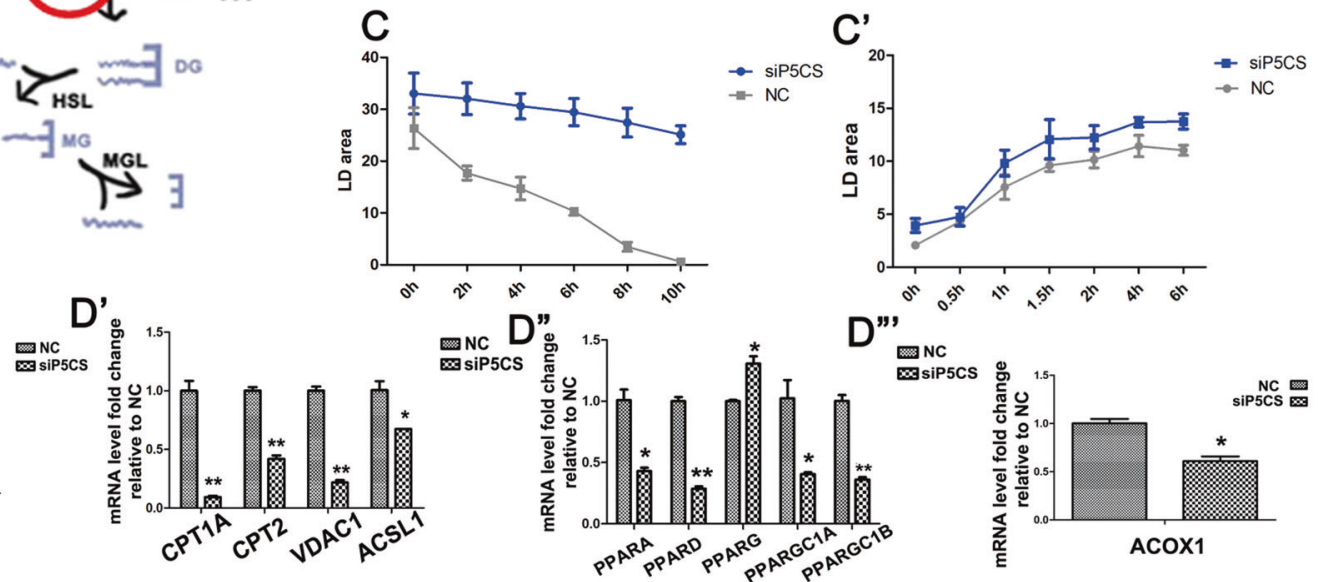

D"

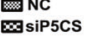

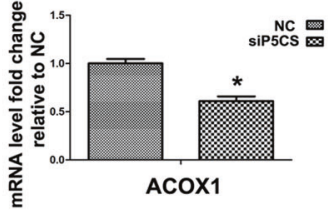

E
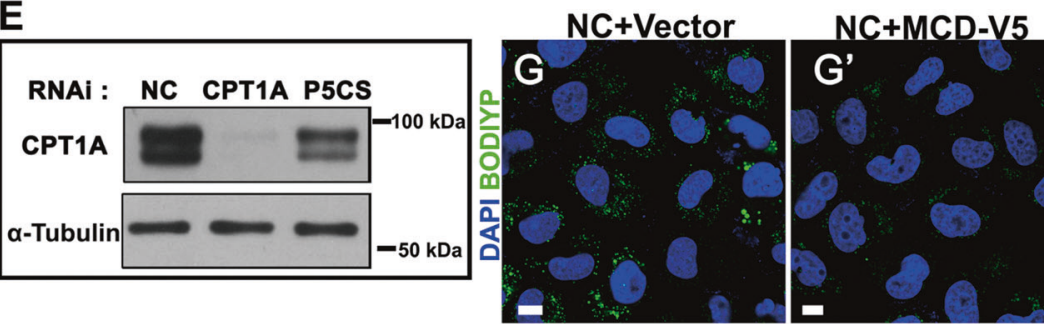

siP5CS+Vector

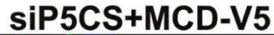

$\mathbf{F}$

$\mathbf{H}$

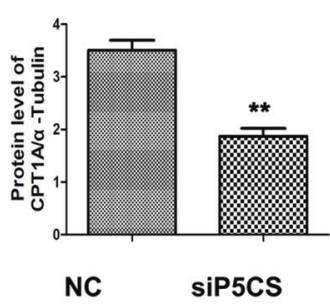

Fig. 6 P5CS loss inhibits fatty acid $\beta$-oxidation. a A diagram showed the key enzymes in TAG synthesis and lipolysis pathway. b A scheme showed the experimental strategies to measure the consumption of LDs. HeLa cells treated with DGAT1 and DGAT2 inhibitors $24 \mathrm{~h}$ after mock treatment or P5CS knockdown. Stained with BODIPY and observed at different time points after the drug treatment. $\mathbf{c}$ The consumption of LDs is decreased in P5CS-deficient cells. $\mathbf{b}^{\prime}$ A scheme showed the experimental strategies to measure the rate of lipid-droplet formation. HeLa cells treated with DGAT1/2 inhibitors when P5CS RNAi knockdown was initiated. Twenty-four hours later, the inhibitors were washed off and an inhibitor of ATGL (ATGLi) was added and LDs were monitored at different time points by BODIPY staining. $\mathbf{c}^{\prime}$ The rates of lipid-droplet formation were largely the same in the control and P5CS knockdown cells. d-d"' Quantitative PCRs (qPCRs) were performed for the indicated genes in control and P5CS RNAi knockdown cells. The expression data are represented as mean
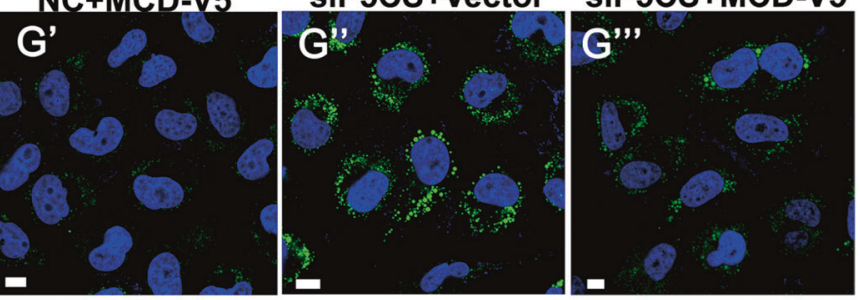

\section{P5Cs KO P5CS KO +proline J}
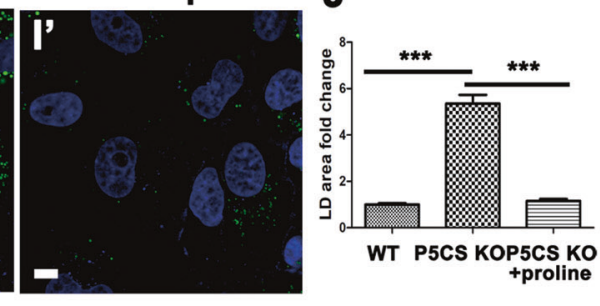

acid $\beta$-oxidation was reduced in P5CS knockdown cells. We confirmed the expression of these genes by quantitative PCR (qPCR). Consistent with the lipid accumulation $\pm \mathrm{SEM}, n=3$. e Western blot results showed that the protein level of CPT1A was reduced in P5CS knockdown cells. $\mathbf{f}$ is the quantification of CPT1A protein level in P5CS knockdown cells. Data were represented as mean + SEM, $n=3$. $\mathbf{g}-\mathbf{g}^{\prime \prime \prime}$ HeLa cells with indicated treatments were stained with BODIPY and DAPI. MCD overexpression partially rescue the accumulation of lipid droplets in P5CS knockdown cells. $\mathbf{h}$ is the quantification of the fold change of the area of LDs in the cells with indicated treatments. Data were represented as mean + SEM, $n=5$ groups (more than 100 cells for each treatment were analyzed). $\mathbf{i}, \mathbf{i}^{\prime}$ P5CS KO cells cultured with or without addition of proline in the culture medium were stained with BODIPY and DAPI. The addition of proline reduced LD accumulation in P5CS KO cells. $\mathbf{j}$ is the quantification of the fold change of the area of LDs in the cells with indicated treatments. Data were represented as mean $+\mathrm{SEM}, n=$ 5 groups (more than 100 cells for each treatment were analyzed).

phenotypes, the expression of genes involved in fatty acid transport (CPT1A and CPT2), fatty acid $\beta$-oxidation (ETFDH, HADH, and ACACB), lipid metabolism related 
A

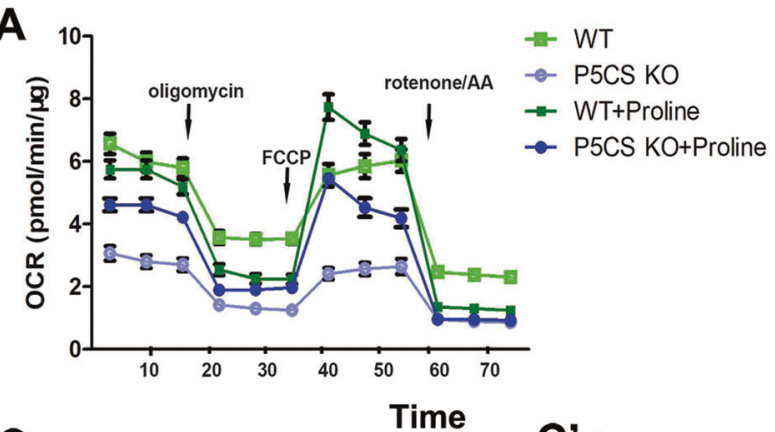

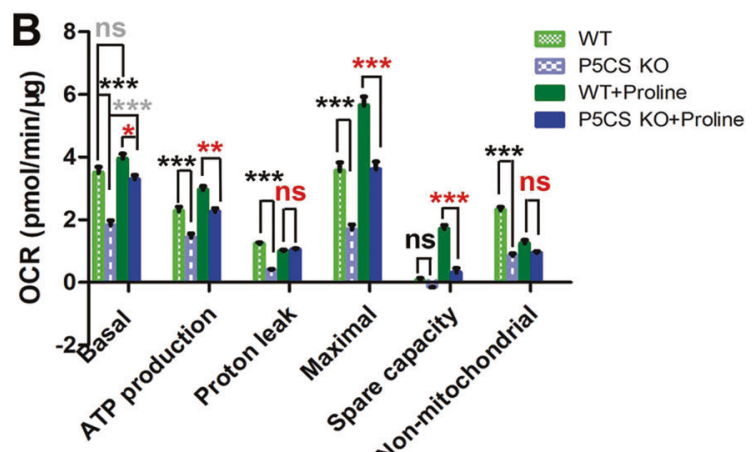
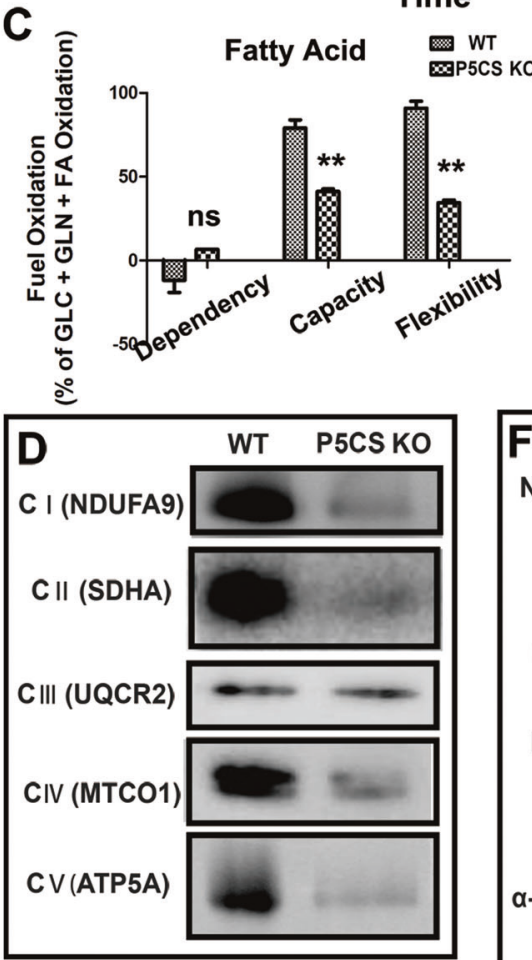

E
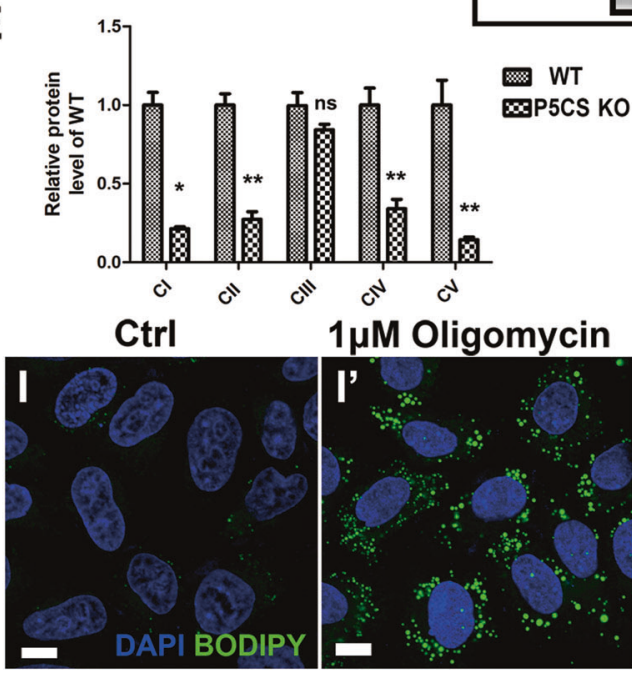

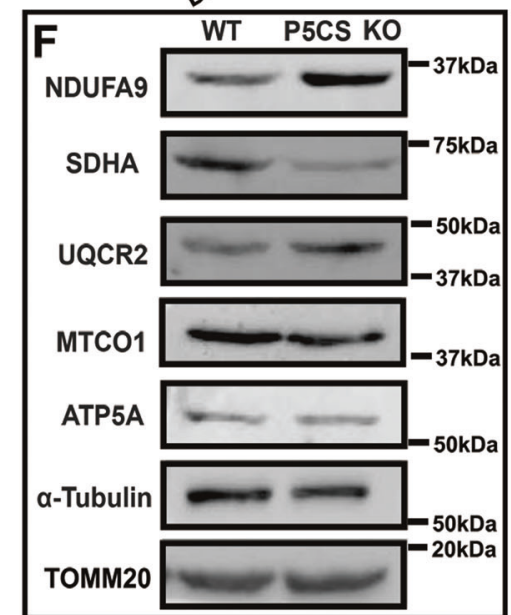

.5uM Rotenone

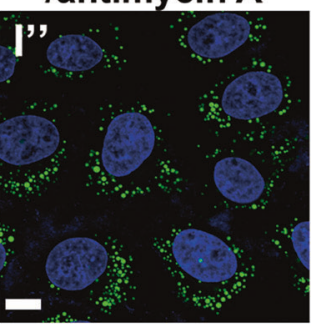

Glutamine $\underset{0}{\mathrm{~W} P \mathrm{PCS}} \mathrm{Wo}$
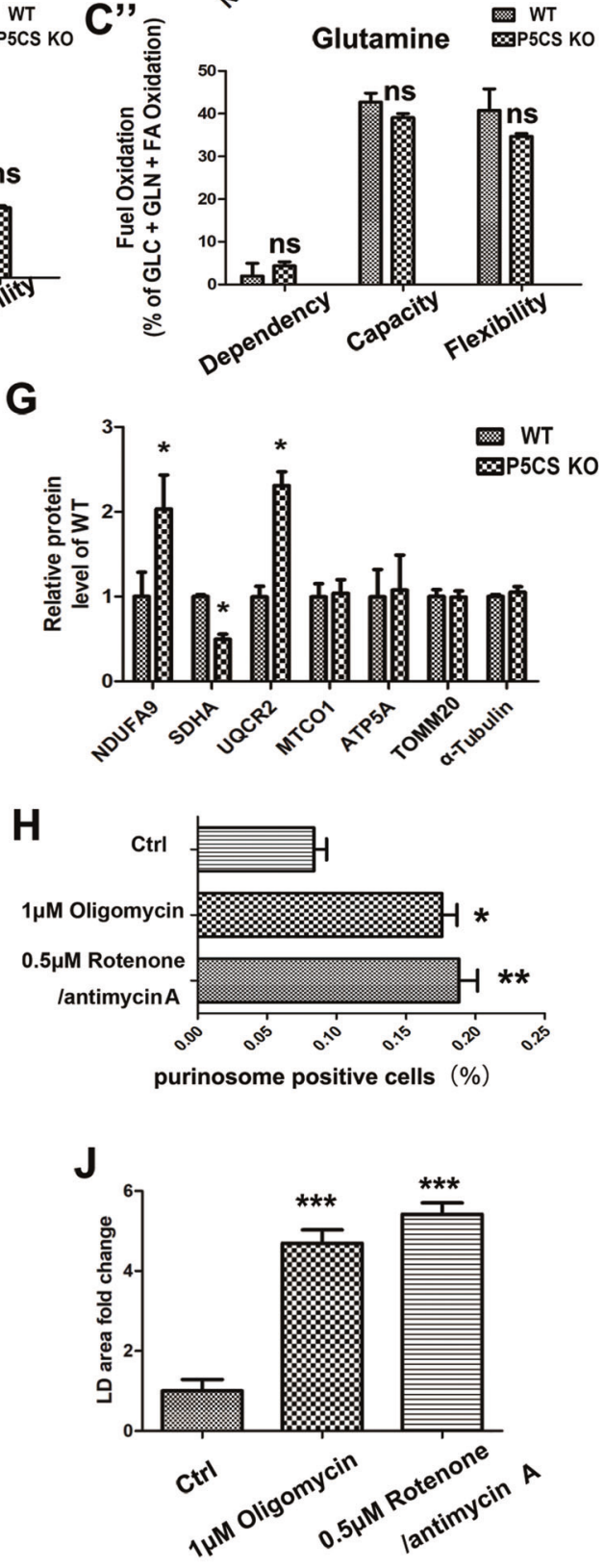

transcription factors (PPARA and PPARD), and PPAR $\gamma$ coactivator (PPARGC1A and PPARGC1B) were all reduced (Fig. 6d-d"'). Western blot results also show in addition to lower mRNA levels, the protein level of CPT1A was also reduced in P5CS knockdown cells (Fig. 6e, f). When we overexpressed MCD, the activator 
Fig. $7 \mathrm{P5CS}$ is required for oxidative respiration in mitochondria. a, b The oxygen consumption rate (OCR) was measured by Seahorse. Basal respiration, ATP production, and maximal respiration were greatly reduced in P5CS KO cells. The OCR defects were partially rescued by the addition of proline to the culture medium. $\mathbf{c}-\mathbf{c}^{\prime \prime}$ The dependency, capacity, and flexibility of cells to oxidize long-chain fatty acids, glucose, and glutamine were determined by measuring mitochondrial OCR in the presence or absence of metabolic pathway inhibitors. The cell's dependency on glucose but not on fatty acid or glutamine was increased when P5CS was deficient. The capacity of the P5CS KO cells using fatty acids as fuel to meet energy defects was reduced. The capacity of P5CS KO cells using glucose and glutamine did not change. Data are presented as means + SEM, $n=3$. d The organization of respiratory complexes was analyzed by BN-PAGE. Complexes were examined by immunoblots with indicated antibodies followed blue native gel separation. The organization of complex I, II, IV, and V are reduced in P5CS KO cells. e Statistical analysis of $\mathbf{d}$. Data were presented as mean $+\mathrm{SEM}, n=3$. $\mathbf{f}$ The protein levels of indicated subunits of respiration complexes were analyzed by western blot. $\alpha$-Tubulin and TOMM20 functioned as loading control. g Statistical analysis of $\mathbf{e}$. Data were presented as mean + SEM, $n=3$. $\mathbf{h}$ Inhibition of mitochondrial respiration by treatments with oligomycin or Rotenone together with antimycin A results in increased purinosome formation, which phenocopies the lack of P5CS. i-j HeLa cells with indicated treatments were subjected to staining with BODIPY and DAPI. The respiration complex inhibitors induced lipid-droplet accumulation. $\mathbf{j}$ is the quantification of the fold changes of LD area in $\mathbf{e}$. Data were presented as mean $+\mathrm{SEM}, n=5$ groups (more than 100 cells for each treatment were analyzed).

of CPT1 [26], we could partially rescue the accumulation of LDs in P5CS knockdown cells (Fig. 6g-g'", h).

\section{P5CS is required for oxidative respiration in mitochondria}

Mitochondria are metabolic centers for lipid $\beta$-oxidation and purine metabolism, and P5CS is a mitochondriallocalized enzyme. We wondered whether the lack of P5CS led to mitochondrial defects that resulted in metabolic abnormalities. Similar to the TEM study, we did not observe any obvious morphology defects in P5CS KO cells when we examined mitochondria through MitoTracker staining. JC-1 staining did not show any mitochondrial membrane potential defects in P5CS KO cells. The ROS production in mitochondria and cytosol indicated by MitoSox and DCFH-DA staining also did not change in P5CS KO cells (Fig. S7). Although there were no obvious mitochondrial morphology defects, the oxygen consumption rate (OCR) was greatly reduced in P5CS KO cells when measured with the Seahorse instrument. Basal respiration, ATP production, and maximal respiration in P5CS KO cells were all greatly reduced (Fig. 7a, b). Overexpression of wild-type P5CS but not $\mathrm{P} 5 \mathrm{CS}^{\mathrm{R} 138 \mathrm{Q}}$ or the enzyme dead form of P5CS (P5CS ${ }^{\mathrm{CD}}$ ) in P5CS KO cells rescued both the LD accumulation and the defects in basal respiration and ATP production, suggesting that enzymatic activity of P5CS might be critical for its function in lipid metabolism and respiration (Fig. S8A-G). Surprisingly, overexpression of either form of P5CS in P5CS KO cells significantly increased the maximal respiration, spare capability, and non-mitochondrial respiration (Fig. S8G), indicating that the overexpressed P5CS can modulate some aspects of respiration independent of its sub-mitochondrial localization and enzymatic activities. Interestingly, when we added proline to the culture medium, the number of LDs was greatly reduced in P5CS KO cells with or without mutant forms of P5CS expression (Figs. 6i-j and S8A-F). Similarly, the OCR defects were partially rescued by the addition of proline (Figs. 7a, b and S8G). Since P5C is the precursor for both proline and ornithine, we also added ornithine to the cultures of P5CS KO cells. The addition of ornithine partially rescued the defects of lipid metabolism and respiration in P5CS KO cells (Fig. S8H-K), suggesting that the rescue effects is not specific to proline.

We then analyzed metabolite fuel usage in the control and P5CS KO cells. The dependency, capacity, and flexibility of cells to oxidize long-chain fatty acids, glucose, and glutamine were determined by measuring mitochondrial OCR in the presence or absence of metabolic pathway inhibitors. Sequential inhibition of the pathway of interest and other metabolic pathways, followed by measurement of OCR provides an indication of how dependent the basal OCR is on the pathway of interest. Dependency indicates that mitochondria are unable to compensate for the blocked pathway by oxidizing other fuels. We found that the cell's dependency on glucose but not on fatty acid or glutamine was increased when P5CS was deficient. To measure the capacity of cells using a specific metabolite, two alternative pathways were first inhibited; this was then followed by inhibition of pathway of interest. Consistent with the reduction of fatty acid $\beta$ oxidation in P5CS cells, the capacity of these cells using fatty acids as fuel to meet energy defects was reduced. The capacity of P5CS KO cells to meet energy demands using glucose and glutamine did not change. Flexibility is calculated by subtracting the fuel dependency from the fuel capacity for the pathway of interest (Fig. $\left.7 \mathrm{c}-\mathrm{c}^{\prime \prime}\right)$. Performing this calculation revealed that P5CS KO cells had reduced flexibility to use fatty acids as fuel.

To understand the respiration defects in P5CS KO cells, we examined the organization of respiratory complexes by blue native $(\mathrm{BN})$ gel and western blot [27]. The $\mathrm{BN}$ gel results indicated that the organization of complex I, II, IV, and $\mathrm{V}$ are reduced in P5CS KO cells (Fig. 7e, f). When we examine the level of the components of these complexes by western blot, only SDHA, the component of complex II, was slightly reduced. Complex I component NDUFA9 and complex III component UQCR2 were even slightly increased (Fig. $7 \mathrm{~g}$ ). These data suggest that the lack of P5CS has an impact on respiratory complex organization.

It has been reported that inhibition of mitochondrial respiration results in increased purinosome formation 
[28, 29]. Indeed, addition of oligomycin or Rotenone together with antimycin A increased the number of purinosome-positive cells cultured in normal medium [28] (Fig. 7h), which phenocopies the lack of P5CS. In addition, treatment with these respiration complex inhibitors also induced LD accumulation in cultured cells (Fig. 7i-i" $\mathrm{i}^{\prime \prime}$ j). These data suggest that metabolic defects in P5CS KO cells are due to defects in mitochondrial respiration.

\section{Discussion}

In plants, the transcription or the activity of P5CS is upregulated and proline accumulates in response to environmental stresses such as drought, high salt, malnutrition, or UV radiation [5]. In this study, we found that P5CS formed rod- or ring-like structures and became diffusely localized in mitochondria in response to oxidative stress caused by starvation or other treatments. Although diseaserelated mutations $\mathrm{P} 5 \mathrm{CS}^{\mathrm{R} 84 \mathrm{Q}}, \mathrm{P} 5 \mathrm{CS}^{\mathrm{G} 93 \mathrm{R}}$, and $\mathrm{P} 5 \mathrm{CS}^{\mathrm{R} 138 \mathrm{Q}}$ somewhat lost the rod- and ring-like patterns in mitochondria, we are currently unable to conclude whether P5CS is more active in the rod- and ring-like structures than in the diffused conditions. Structural studies will provide more insight in this regard. Interestingly, we demonstrated that P5CS senses and responds to cellular stress in mammalian cells. Cells without P5CS are sensitized to HBSS culture conditions, suggesting that P5CS might function as a sensor or modulator in response to stress. CL patients with P5CS mutations have premature aging features, such as hyper-elastic skins and cataracts that also occur during normal aging process [30]. We found that P5CS became diffusely localized in mitochondria in senescent cells. It will be worthwhile to investigate whether changes in P5CS localization contribute to the changes in cellular and tissue features that are observed during the normal aging process.

In this study, we found that lack of P5CS also led to reduced oxidative respiration. It is not clear whether P5CS participates in organization of respiratory complexes directly or whether it functions through altering metabolite flux. Interestingly, adding proline to the culture medium could partially rescue the respiration defects, suggesting that proline (or products of its metabolism) might participate in the organization of respiratory complex. Proline has been reported to function as a chaperone in order to diminish protein aggregates [31]. Further investigation is required to determine whether proline acts as a chaperone to regulate oxidative respiration. We found that the rescue effects were not limited to the addition of proline. The addition of ornithine also partially rescued the defects in P5CS KO cells. It is not surprising since proline and ornithine can be converted to P5C, or converted to each other in P5CS KO cells. It is not clear whether a reduction of P5C, proline, or ornithine, directly contributes to the phenotypes. Overexpression of catalytic activity dead form of P5CS affected the maximal respiration, spare capability, and nonmitochondrial respiration, suggesting that P5CS might play other roles beyond P5C synthesis in order to maintain proper oxidative respiration.

We found that loss of P5CS affected pathways beyond proline- and ornithine-related metabolism. The lipid and purine metabolic pathways were also affected, suggesting that multiple metabolic pathways are interconnected in mitochondria. This may explain the complexity of disease symptoms in patients with P5CS mutations. Reduced oxidative respiration leads to reduced lipid consumption [22] and increased purinosome formation [28], which contributed to the metabolic defects we observed in P5CS KO cells. However, we cannot exclude the possibility that some phenotypes may be due to compensatory effects of increasing or reducing the levels of certain metabolites.

In flies, the lack of P5CS results in a reduced eclosion rate and neurodegeneration. The increase LDs in the eyes of young P5CS KO flies might contribute to eye degeneration, as reported previously [22]. It would be interesting to test whether patients with SPG also have ectopic LD accumulation. Furthermore, it would also be worth evaluating whether there are mitochondrial respiration defects in the cells isolated from those patients.

\section{Methods}

\section{Plasmids and siRNAs}

P5CS-V5 and MCD-V5 plasmids were constructed by inserting full-length cDNA of P5CS and MCD into pcDNA3.1(+) vector, respectively, V5 tag was fused at the Ctermini of the proteins. P5CS-Myc plasmid was constructed by inserting full-length P5CS cDNA into pcDNATM 3.1MycHis(-)A vector. All mutated forms of P5CS were obtained through site-directed mutagenesis. Truncation forms of P5CS were generated based on P5CS-V5 plasmid. To construct TOMM20-Apex construct, cDNA encoding TOMM20 and Apex (Addgene, \#67651) were inserted into pEGFPN1 vector. To generate P5CS-linker-Apex-V5 construct, cDNA encoding P5CS, a linker sequence GSGGSGSGSSGGSGS, and Apex were inserted into pEGFPN1 vector, V5 tag was introduced by PCR amplification. pFGAMS-mEos was purchased from Addgene (\#105128).

The siRNA sequences used were as follows:

P5CS: 5'-CCCUGGGUCAAGUGUACAATT- $3^{\prime}$.

5'-AUGAAAAGCCUGUGCGUGATT- 3 '.

CPT1A: 5'-CCAUGGAUCUGCUGUAUAUTT- $3^{\prime}$. CPT2A: 5'-CCAGGCUGCCUAUUCCCAATT-3'. 
SLC25A20: 5'-GAACGGAUCAAGUGCUUAUTT-3'. ACADL: 5'-GCUCAGAAGAACAGAUUAATT-3'. ACAD9: 5'-GCAAGUCCCAGAAGAAUAUTT-3'. GSS: 5'-GCAGGAUAAACAGCAGCUATT-3'. GSR: 5'-GCAAGUCCCAGAAGAAUAUTT-3'. GCGL: 5'-GGAGGAAACCAAGCGCCAUTT-3'.

\section{Antibodies and reagents}

The following primary antibodies were used: Anti-TOMM20 antibody (BD Biosciences, 612278); anti-HSP60 antibody (Cell Signaling, 12165); anti-HA antibody (Cell Signaling, 3724); anti-Myc antibody (Santa Cruz, sc-40); anti-V5 antibody (Life Technologies, 46-0705); anti- $\alpha$-tubulin antibody (Boster, BM1452); anti-NDUFA9 antibody (Abcam, ab14713); anti-SDHA antibody (Abcam, ab14715); antiUQCRC2 antibody (Abcam, ab14745); anti-MTCO1 antibody (Abcam, ab14705); anti-ATP5A antibody (Abcam, ab14748); anti-CPT1A antibody (Abcam, ab128568); anti-P21 antibody (Abcam, ab109520); anti-ACTB antibody (Abcam, ab8226); anti- $\gamma \mathrm{H} 2 \mathrm{AX}$ antibody (Cell Signaling, 9718S); and anti-P5CS antibody (Sigma, HPA012604). DCFH-DA and CCK-8 Kit was from Beyotime. JC-1, BODIPY 493/403, MitoTracker Green, MitoSOX, Lipofectamine 2000, and Lipofectamine RNAi MAX were from Life Technologies. NAC (10 mM), Bafilomycin A1 $(250 \mathrm{nM})$, T863 (DGAT1i) $(20 \mu \mathrm{M})$, PF-06424439 (DGAT2i) $(10 \mu \mathrm{M})$ were purchased from Sigma. ATGListatin $(20 \mu \mathrm{M})$ was from EMD Millipore (\#5.30151.0001). Etomoxir $(100 \mu \mathrm{M})$ was from Cayman Chemical (\#11969). Mito-TEMPO (10 $\mu \mathrm{M})$ was from Sigma.

\section{Animals}

The Drosophila stocks were obtained from the Bloomington Drosophila Stock Center. The CRISPR/Cas9 KO flies were generated as described before [32]. Briefly, germline specific Cas9, Nos-Cas9 stock (67083), was crossed to CG7470 TRiP-KO stock (76969). The male F1 progeny containing both Nos-Cas9 and sgRNA targeting CG7470 transgenes were crossed to the balancer stocks. The male F2 progeny were collected and crossed individually with balancer stocks to establish the mutant stocks. Once the offspring started to emerge, the male flies were picked out and sequenced to identify the potential mutants. Animals used in the experiments were randomly chosen for each genotype. The genotypes were known for the researchers during the experiments.

\section{Cell culture, transfection, and generation of CRISR/ PCas9 KO cell lines}

HeLa, U2OS, and HEK293T cells were originally from ATCC and were recently authenticated and tested for contamination. Cells were cultured in DMEM supplemented with $10 \%$ fetal bovine serum and $1 \%$ penicillinstreptomycin, at $37{ }^{\circ} \mathrm{C}$ with $5 \% \mathrm{CO}_{2}$. To induce senescence, IMR-90 cells were incubated with $100-\mathrm{ng} / \mathrm{ml}$ Doxorubicin (Selleck) for $12 \mathrm{~h}$. Then the cells were released to normal condition and cultured for a week. For transfection, cells were transfected with constructs needed using Lipofectamine 2000 according to the manufacturer's instructions. Similarly, cells were transfected with appropriate siRNAs by using Lipofectamine RNAi MAX according to the manufacturer's instructions. The knockdown efficiencies were confirmed by RT-PCR. The P5CS KO cell lines were generated by type-II CRISPR nuclease system as described previously (Cong et al., Science 2013). The cell lines used were confirmed by sequencing and western blot. For amino acid starvation, cells were cultured in DMEM (D9800-13, US Biological) supplemented with 4.5-g/L glucose, and $10 \%$ FBS. For glutamine starvation, DMEM (A1443001, Life Technologies) was supplemented with 10\% FBS, 4.5$\mathrm{g} / \mathrm{L}$ glucose and $10 \% \mathrm{FBS}, 1 \mathrm{~g} / \mathrm{L}$ glucose. For low-glucose conditions, DMEM (A1443001, Life Technologies) was supplemented with $10 \%$ FBS, $4.5-\mathrm{g} / \mathrm{L}$ glucose and $10 \%$ FBS, and 4-mM glutamine. For proline rescue, 2-mM proline were supplemented $24 \mathrm{~h}$ before test.

\section{Cell proliferation assay}

Cell viability was detected via a cell counting kit-8 (CCK-8, Beyotime) assay. Wild-type control and P5CS KO cells were plated at $3 \times 10^{4}$ cells per well in 96-well plates, $48 \mathrm{~h}$ after plating, CCK-8 solution $(10 \mu \mathrm{L})$ was added into each well and incubated for $4 \mathrm{~h}$. Cell proliferation was calculated through measuring the absorbance at $450 \mathrm{~nm}$ by microplate reader. Three replicates were included per condition, and experiments were repeated three times with means used for experimental analyses.

\section{RNA isolation and real-time PCR (qPCR)}

RNA from cells was harvested using TRIzol (Thermo Fisher Scientific) according to manufacturer's protocol, and then converted into cDNA using M-MLV Reverse Transcriptase (Invitrogen) according to the manufacturer's instructions. Quantitative qPCR reactions were performed using Trans Start Green qPCR Super Mix (TransGen Biotech) and a BioRad CFX96 Touch RT-PCR Detection System. The primers used could be found in the Supplemental Table S4.

\section{PK treatment and alkaline carbonate extraction}

PK treatment and alkaline carbonate extraction were performed as described previously [33]. 


\section{Co-immunoprecipitation}

Forty-eight hours after transfection, cells were harvested and resuspended in $600-\mu \mathrm{L}$ ice cold lysis buffer $(50 \mathrm{mM}$ Tris, $150 \mathrm{mM} \mathrm{NaCl}, 1 \mathrm{mM}$ EDTA and $1 \%$ TritonX-100, $\mathrm{pH}$ 7.4) for $30 \mathrm{~min}$ on ice. The lysates were centrifuged at $13,000 \mathrm{rpm}$ for $10 \mathrm{~min}$ at $4{ }^{\circ} \mathrm{C}$. Sixty microliters supernatants were saved as input. The rest were transferred to a clean eppendorf tube and incubated $6 \mathrm{~h}$ at $4{ }^{\circ} \mathrm{C}$ with $10-\mu \mathrm{L}$ anti-Myc-beads (Sigma). The beads were washed three times in lysis buffer before boiling in loading buffer. Western blotting was then performed.

\section{BN-PAGE analysis of mitochondrial complexes}

BN-PAGE was conducted using the NativePAGETM system (Invitrogen). Briefly, mitochondria were isolated from control and P5CS KO HeLa cells as described before [33]. The isolated mitochondria were solubilized by $10 \%$ lauryl maltoside solution (n-dodecyl- $\beta$-D-maltopyranoside, ab109857) for $30 \mathrm{~min}$ on ice, then centrifuged at 20,000 $\mathrm{g}$ at $4{ }^{\circ} \mathrm{C}$ for $30 \mathrm{~min}$. After centrifugation, the supernatants were collected and the protein concentration was determined by BCA analysis (ThermoFisher). Add 1/20 5\% solution/suspension of Coomassie blue G-250 (Invitrogen) in $0.5-\mathrm{M}$ aminocaproic acid to the supernatant and then loaded equivalent protein of control and P5CS KO samples into a 3-12\% non-denaturing polyacrylamide gel (Invitrogen). After electrophoresis, proteins were transferred to a PVDF membrane and then probed with specific antibodies against subunits of complex I (NDUFB9), complex II (SDHA), complex III (UQCRC1), complex IV (COX IV), and complex V (ATP5A). Blots were visualized using secondary antibodies conjugated with horseradish peroxidase.

\section{Mitochondrial respiration measurements}

Briefly, mitochondrial respiration-related assays were conducted with the Seahorse XFp Mito Fuel Flex Test Kit (103270-100) and Seahorse XFp Cell Mito Stress Test Kit (103010-100) using Seahorse XFp Flux Analyzer (Seahorse Bioscience) according to the manufacturer's instructions. Briefly, about $1.5 \times 10^{4}$ control and P5CS KO HeLa cells overexpressed with indicated plasmids were seeded onto XFp microplate the day before experiment. After $24 \mathrm{~h}$, cells were incubated in DMEM for $24 \mathrm{~h}$ in the presence or absence of 2-mM proline/ornithine. The OCRs were measured and the averages from three independent experiments were calculated. For mito-stress test, Oligomycin $(10 \mu \mathrm{M})$, FCCP $(2.5 \mu \mathrm{M})$, and rotenone/antimycin $(5 \mu \mathrm{M})$ were injected at the indicated time points. The mean \pm SEM was determined and statistical significance was evaluated using the Student $t$ test with a $P$ value $<0.05$. The OCRs were average from three independent experiments and the protein content of each well was then measured to normalize OCR values.

\section{Immunofluorescence and confocal microscopy}

For immunostaining, cells grown on glass coverslips were fixed in $4 \%$ formaldehyde for $20 \mathrm{~min}$ before they were permeabilized with $0.1 \%$ Triton $\mathrm{X}-100$ for $10 \mathrm{~min}$ and blocked with $3 \%$ bovine serum albumin for $30 \mathrm{~min}$. The cells were then incubated with appropriate primary antibodies at $4{ }^{\circ} \mathrm{C}$ overnight followed by washing with PBS thoroughly. Then the cells were stained with fluorescent secondary antibodies for $1 \mathrm{~h}$ at room temperature. Nuclei were stained with $1 \mu \mathrm{g} / \mathrm{mL}$ DAPI in PBS for $5 \mathrm{~min}$. After washing, the samples were mounted for confocal microscopy.

DCFH-DA and JC-1 staining were performed as described previously [33]. LDs were stained with $0.2-\mu \mathrm{g} /$ $\mathrm{mL}$ BODIPY 493/503 (Thermo Fisher Scientific) in PBS with $1 \%$ bovine serum albumin for $5 \mathrm{~min}$ at room temperature. For MitoSOX staining and MitoTracker staining, $5 \mu \mathrm{M}$ of MitoSOX or $100 \mathrm{nM}$ of MitoTracker Green and were loaded into cells at $37^{\circ} \mathrm{C}$ for $30 \mathrm{~min}$ followed by three washes with Tyrode's solution. Fluorescence images were acquired on Zeiss 710 confocal microscope and GE DeltaVision OMX.

SA- $\beta$-galactosidase activity were measured by using SA$\beta$-gal kit (Cell signaling Technology) according to manufacturer's protocol.

\section{Transmission electron microscopy}

For Drosophila eyes, the fly heads were dissected and fixed at $4{ }^{\circ} \mathrm{C}$ in $2 \%$ paraformaldehyde (Electron Microscopy Sciences, 15710); 2\% glutaraldehyde (Electron Microscopy Sciences, 16020); 0.1-M sodium cacodylate pH 7.2 (Electron Microscopy Sciences, 12201). Then, they were post fixed in 2\% OsO4 (Electron Microscopy Sciences, 19152). The 50-nm thin sections were stained with $4 \%$ uranyl acetate (Electron Microscopy Sciences, 22400) and 2.5\% lead nitrate (Electron Microscopy Sciences, 17800) for electron microscopy analysis (Hitachi Ltd, HT7700, Tokyo, Japan). TEM for mammalian cells was performed as described previously [33] using a Hitachi HT7700 electron microscope. For APEX labeling, the cells were processed based on a protocol previously reported by Ariotti et al. [34]. Instead of co-expression interest protein with GFP tag and APEX-GBP, we directly fused our target proteins with Apex tags. 


\section{Metabonomics}

The metabonomics analysis was performed with assistance of Novogene Inc.

\section{Sample preparation}

Sample preparation for metabolomic followed a protocol reported by Sellick et al. [35]. Briefly, the culture medium was discarded and the cells were washed with pre-cooled PBS for 2-3 times. The cells were scraped off with $1 \mathrm{~mL}$ pre-cooled $60 \%$ methanol solution (Sigma, chromatographic level) and centrifuged at $1000 \mathrm{~g}$ at $4{ }^{\circ} \mathrm{C}$ for $1 \mathrm{~min}$. The precipitate was transferred into cryovial tubes and stored at $-80^{\circ} \mathrm{C}$ after liquid nitrogen frozen. Transferred $50-\mu \mathrm{L}$ cell samples to a new tube, added $200-\mu \mathrm{L} 80 \%$ methanol aqueous solution and ultrasound $6 \mathrm{~min}$, centrifuged at $15,000 \mathrm{~g}$ at $4{ }^{\circ} \mathrm{C}$ for $10 \mathrm{~min}$. Took supernatant and added $1 / 2$ volume of mass spectrometry grade water to dilute to $53 \%$ methanol content. Centrifuged them at $15,000 \mathrm{~g}$ at $4{ }^{\circ} \mathrm{C}$ for $20 \mathrm{~min}$ and collected the supernatant for LC-MS analysis.

\section{LC-MS analysis for cells metabolome}

The LC systems used were QTRAP6500+(SCIEX) and Exion LC(SCIEX). For positive ion mode: LC separation was conducted on $\mathrm{C} 8100 \times 2.1 \mathrm{~mm}$ column. Mobile phases A $(0.1 \%$ formic acid $)$ and B ( $0.1 \%$ formate-acetonitrile) were used to develop a gradient elution. The gradient program was as following: $0-1 \mathrm{~min}, 95 \% \mathrm{~A}+5 \% \mathrm{~B} ; 24-28$ min, linear gradient to $0 \% \mathrm{~A}+100 \% \mathrm{~B} ; 28-30 \mathrm{~min}, 95 \%$ $\mathrm{A}+5 \% \mathrm{~B}$; flow rate, $0.35 \mathrm{~mL} / \mathrm{min}$. The column oven temperature was maintained at $50{ }^{\circ} \mathrm{C}$. For negative ion mode: LC separation was conducted on $\mathrm{T} 3100 \times 2.1 \mathrm{~mm}$. Mobile phases A (6.5-mM ammonium bicarbonate) and B (6.5-mM ammonium bicarbonate-95\% methanol) were used to develop a gradient elution. The gradient program was as following: $0-1 \mathrm{~min}, 98 \% \mathrm{~A}+2 \% \mathrm{~B} ; 18-22 \mathrm{~min}$, linear gradient to $0 \% \mathrm{~A}+100 \% \mathrm{~B} ; 22-25 \mathrm{~min}, 95 \% \mathrm{~A}+$ $5 \% \mathrm{~B}$; flow rate, $0.35 \mathrm{~mL} / \mathrm{min}$. The column oven temperature was maintained at $50^{\circ} \mathrm{C}$.

\section{Data analysis}

The experimental samples were analyzed based on Novogene database, multi-reaction monitoring mode. The compounds were quantified according to Q3(sub-ions) and qualitatively analyzed by Q1 (parent ion), Q3 (sub-ion), RT (retention time), DP (declustering voltage), and CE (collision energy). The SCIEX OSV1.4 software was used to open the off-machine mass spectrum file for the chromatographic peak integration and correction work.

\section{RNA isolation and RNA sequencing}

RNA isolation and RNA sequencing were performed as described previously [36]. Briefly, total RNA was extracted from approximately one million of NC and P5CS RNAi HeLa cells using a RNeasy Plus Mini kit (QIAGEN). The sequencing library was constructed following the manufacturer's protocol using NEBNext kits (E7490). Highthroughput sequencing was performed on illumina Hiseq2500 and gene expression were analyzed through TopHat (v2.0.11).

\section{Statistical analysis}

For fluorescence microscopy, images were thresholded, the area of BODIPY 493/503 stained LDs were quantified from three independent experiments (average of over 100 cells in five random images per experiment). For immunoblots, band density quantified using ImageJ software. Mean + SEM was determined from three independent experiments. Comparisons between two groups were assessed by Student $t$ test using Prism 5 software (GraphPad Software). Comparisons over two groups were assessed by one-way ANOVA with Bonferroni multiple comparisons test as post hoc analysis. ${ }^{*} P<0.05, * * P<0.001, * * * P<0.0001$.

Acknowledgements We are grateful to THFC, BDSC, and DGRC for providing fly strains and cDNA clones. We thank the imaging core facilities and mass spectrometry facilities in LSI for the technical support. CT is supported by National Natural Science Foundation of China (91754103, 31622034, 31571383), National Key Research \& Developmental Program of China (2017YFC1001500, 2017YFC1001100), Natural Science Foundation of Zhejiang Province, China (LR16C070001) and Fundamental research funds for the central universities. CT is a Qianjiang Scholar.

Author contributions CT, ZY, XZ, YL, J-FJ, and J-PL designed and performed experiments, analyzed data, and wrote the paper. CT obtained financial support and were responsible for the study design and interpretation of results. WS performed the TEM analysis. All authors approved the final version of the manuscript.

\section{Compliance with ethical standards}

Conflict of interest The authors declare that they have no conflict of interest.

Publisher's note Springer Nature remains neutral with regard to jurisdictional claims in published maps and institutional affiliations.

\section{References}

1. Martinez-Reyes I, Chandel NS. Mitochondrial TCA cycle metabolites control physiology and disease. Nat Commun. 2020;11:102.

2. Spinelli JB, Haigis MC. The multifaceted contributions of mitochondria to cellular metabolism. Nat cell Biol. 2018;20:745-54. 
3. Kauppila TES, Kauppila JHK, Larsson NG. Mammalian mitochondria and aging: an update. Cell Metab. 2017;25:57-71.

4. Sun N, Youle RJ, Finkel T. The mitochondrial basis of aging. Mol Cell. 2016;61:654-66.

5. Perez-Arellano I, Carmona-Alvarez F, Martinez AI, RodriguezDiaz J, Cervera J. Pyrroline-5-carboxylate synthase and proline biosynthesis: from osmotolerance to rare metabolic disease. Protein Sci: Publ Protein Soc. 2010;19:372-82.

6. Panza E, Escamilla-Honrubia JM, Marco-Marin C, Gougeard N, De Michele G, Morra VB, et al. ALDH18A1 gene mutations cause dominant spastic paraplegia SPG9: loss of function effect and plausibility of a dominant negative mechanism. Brain: J Neurol. 2016;139:e3.

7. Koh K, Ishiura H, Beppu M, Shimazaki H, Ichinose Y, Mitsui J, et al. Novel mutations in the ALDH18A1 gene in complicated hereditary spastic paraplegia with cerebellar ataxia and cognitive impairment. J Hum Genet. 2018;63:1009-13.

8. Skidmore DL, Chitayat D, Morgan T, Hinek A, Fischer B, Dimopoulou A, et al. Further expansion of the phenotypic spectrum associated with mutations in ALDH18A1, encoding Delta (1)-pyrroline-5-carboxylate synthase (P5CS). Am J Med Genet Part A. 2011;155A:1848-56.

9. Wolthuis DF, van Asbeck E, Mohamed M, Gardeitchik T, LimMelia ER, Wevers RA, et al. Cutis laxa, fat pads and retinopathy due to ALDH18A1 mutation and review of the literature. Eur J Paediatr Neurol: Off J Eur Paediatr Neurol Soc. 2014;18:511-5.

10. Fischer-Zirnsak B, Escande-Beillard N, Ganesh J, Tan YX, Al Bughaili M, Lin AE, et al. Recurrent de novo mutations affecting residue Arg138 of pyrroline-5-carboxylate synthase cause a progeroid form of autosomal-dominant cutis laxa. Am J Hum Genet. 2015;97:483-92.

11. Krishnan N, Dickman MB, Becker DF. Proline modulates the intracellular redox environment and protects mammalian cells against oxidative stress. Free Radic Biol Med. 2008;44:671-81.

12. Shafqat S, Velaz-Faircloth M, Henzi VA, Whitney KD, YangFeng TL, Seldin MF, et al. Human brain-specific L-proline transporter: molecular cloning, functional expression, and chromosomal localization of the gene in human and mouse genomes. Mol Pharmacol. 1995;48:219-29.

13. Wang D, Liu Y, Zhang R, Zhang F, Sui W, Chen L, et al. Apoptotic transition of senescent cells accompanied with mitochondrial hyper-function. Oncotarget. 2016;7:28286-300.

14. Rebbaa A, Zheng X, Chou PM, Mirkin BL. Caspase inhibition switches doxorubicin-induced apoptosis to senescence. Oncogene. 2003;22:2805-11.

15. Perez-Arellano I, Rubio V, Cervera J. Mapping active site residues in glutamate-5-kinase. The substrate glutamate and the feed-back inhibitor proline bind at overlapping sites. FEBS Lett. 2006;580:6247-53.

16. Marco-Marin C, Gil-Ortiz F, Perez-Arellano I, Cervera J, Fita I, Rubio V. A novel two-domain architecture within the amino acid kinase enzyme family revealed by the crystal structure of Escherichia coli glutamate 5-kinase. J Mol Biol. 2007;367:1431-46.

17. Page R, Nelson MS, von Delft F, Elsliger MA, Canaves JM, Brinen LS, et al. Crystal structure of gamma-glutamyl phosphate reductase (TM0293) from Thermotoga maritima at $2.0 \mathrm{~A}$ resolution. Proteins. 2004;54:157-61.

18. Liu ZJ, Sun YJ, Rose J, Chung YJ, Hsiao CD, Chang WR, et al. The first structure of an aldehyde dehydrogenase reveals novel interactions between NAD and the Rossmann fold. Nat Struct Biol. 1997;4:317-26.

19. Wu CA, Chao Y, Shiah SG, Lin WW. Nutrient deprivation induces the Warburg effect through ROS/AMPK-dependent activation of pyruvate dehydrogenase kinase. Biochimica Biophysica Acta. 2013;1833:1147-56.

20. Meister A, Anderson ME. Glutathione. Annu Rev Biochem. 1983;52:711-60.

21. Rambold AS, Cohen S, Lippincott-Schwartz J. Fatty acid trafficking in starved cells: regulation by lipid droplet lipolysis, autophagy, and mitochondrial fusion dynamics. Developmental Cell. 2015;32:678-92.

22. Liu L, Zhang K, Sandoval H, Yamamoto S, Jaiswal M, Sanz E, et al. Glial lipid droplets and ROS induced by mitochondrial defects promote neurodegeneration. Cell. 2015;160:177-90.

23. Harris CA, Haas JT, Streeper RS, Stone SJ, Kumari M, Yang K, et al. DGAT enzymes are required for triacylglycerol synthesis and lipid droplets in adipocytes. J lipid Res. 2011;52:657-67.

24. Nguyen TB, Louie SM, Daniele JR, Tran Q, Dillin A, Zoncu R, et al. DGAT1-dependent lipid droplet biogenesis protects mitochondrial function during starvation-induced autophagy. Developmental Cell. 2017;42:9-21 e25.

25. Mayer N, Schweiger M, Romauch M, Grabner GF, Eichmann TO, Fuchs E, et al. Development of small-molecule inhibitors targeting adipose triglyceride lipase. Nat Chem Biol. 2013;9:785-7.

26. Cuthbert KD, Dyck JR. Malonyl-CoA decarboxylase is a major regulator of myocardial fatty acid oxidation. Curr hypertension Rep. 2005;7:407-11.

27. Konovalova S. Analysis of mitochondrial respiratory chain complexes in cultured human cells using blue native polyacrylamide gel electrophoresis and immunoblotting. J Vis Exp. 2019;144. https://doi.org/10.3791/59269.

28. French JB, Jones SA, Deng H, Pedley AM, Kim D, Chan CY, et al. Spatial colocalization and functional link of purinosomes with mitochondria. Science. 2016;351:733-7.

29. Pedley AM, Benkovic SJ. A new view into the regulation of purine metabolism: the purinosome. Trends Biochem Sci. 2017;42:141-54.

30. Vanakker O, Callewaert B, Malfait F, Coucke P. The genetics of soft connective tissue disorders. Annu Rev Genom Hum Genet. 2015;16:229-55.

31. Ignatova Z, Gierasch LM. Inhibition of protein aggregation in vitro and in vivo by a natural osmoprotectant. Proc Natl Acad Sci USA. 2006;103:13357-61.

32. Ren X, Sun J, Housden BE, Hu Y, Roesel C, Lin S, et al. Optimized gene editing technology for Drosophila melanogaster using germ line-specific Cas9. Proc Natl Acad Sci USA. 2013;110:19012-7.

33. Zhang Y, Liu X, Bai J, Tian X, Zhao X, Liu W, et al. Mitoguardin regulates mitochondrial fusion through MitoPLD and is required for neuronal homeostasis. Mol Cell. 2016;61:111-24.

34. Ariotti N, Hall TE, Rae J, Ferguson C, McMahon KA, Martel N, et al. Modular detection of GFP-labeled proteins for rapid screening by electron microscopy in cells and organisms. Developmental Cell. 2015;35:513-25.

35. Sellick CA, Hansen R, Stephens GM, Goodacre R, Dickson AJ. Metabolite extraction from suspension-cultured mammalian cells for global metabolite profiling. Nat Protoc. 2011;6:1241-9.

36. Xiao Z, Cheng G, Jiao Y, Pan C, Li R, Jia D, et al. Holo-Seq: single-cell sequencing of holo-transcriptome. Genome Biol. 2018;19:163. 